JOURNAL OF THE AMERICAN MATHEMATICAL SOCIETY

Volume 21, Number 1, January 2008, Pages 235-258

S 0894-0347(07)00569-3

Article electronically published on April 25, 2007

\title{
THE STRANGE DUALITY CONJECTURE FOR GENERIC CURVES
}

\author{
PRAKASH BELKALE
}

\section{INTRODUCTION}

Let $S U_{X}(r)$ be the moduli space of semi-stable vector bundles of rank $r$ with trivial determinant over a connected smooth projective algebraic curve $X$ of genus $g \geq 1$ over $\mathbb{C}$. Recall that a vector bundle $E$ on $X$ is called semi-stable if for any subbundle $V, \operatorname{deg}(V) / \operatorname{rk}(V) \leq \operatorname{deg}(E) / \operatorname{rk}(E)$. Points of $S U_{X}(r)$ correspond to isomorphism classes of semi-stable rank $r$ vector bundles with trivial determinant up to an equivalence relation.

For any line bundle $L$ of degree $g-1$ on $X$ define $\Theta_{L}=\left\{E \in S U_{X}(r), h^{0}(E \otimes L) \geq\right.$ 1\}. This turns out be a non-zero Cartier divisor whose associated line bundle $\mathcal{L}=\mathcal{O}\left(\Theta_{L}\right)$ does not depend upon $L$. It is known that $\mathcal{L}$ generates the Picard group of $S U_{X}(r)$ (for this and the precise definition of $\mathcal{L}$ in terms of determinant of cohomology see [DN]).

Let $U_{X}^{*}(k)$ be the moduli space of semi-stable rank $k$ and degree $k(g-1)$ bundles on $X$. Recall that on $U_{X}^{*}(k)$ there is a canonical non-zero theta (Cartier) divisor $\Theta_{k}$ whose underlying set is $\left\{F \in U_{X}^{*}(k), h^{0}(X, F) \neq 0\right\}$. Put $\mathcal{M}=\mathcal{O}\left(\Theta_{k}\right)$. Consider the natural map $\tau_{k, r}: S U_{X}(r) \times U_{X}^{*}(k) \rightarrow U_{X}^{*}(k r)$ given by tensor product. From the theorem of the square, it follows that $\tau_{k, r}^{*} \mathcal{M}$ is isomorphic to $\mathcal{L}^{k} \otimes \mathcal{M}^{r}$. The canonical element $\Theta_{k r} \in H^{0}\left(U_{X}^{*}(k r), \mathcal{M}\right)$ and the Kunneth theorem give a map well defined up to scalars:

$$
H^{0}\left(U_{X}^{*}(k), \mathcal{M}^{r}\right)^{*} \rightarrow H^{0}\left(S U_{X}(r), \mathcal{L}^{k}\right) .
$$

The strange duality conjecture asserts that $(\dagger)$ is an isomorphism. It is known that $h^{0}\left(U_{X}^{*}(k), \mathcal{M}^{r}\right)$ equals $h^{0}\left(S U_{X}(r), \mathcal{L}^{k}\right)$ (see for example [B2], Section 8). This conjecture is known to hold when

- $k=1$ and arbitrary $r$ BNR,

- $r=2, k=2$, and $C$ has no vanishing thetanull B1,

- $r=2, k=4$, and $C$ has no vanishing thetanull vGP,

- $r=2, k$ even, and $k \geq 2 g-4$, and generic $C[\mathrm{~L}$.

An element $F$ of $U_{X}^{*}(k)$ produces an element $\Theta_{F}$ of $H^{0}\left(S U_{X}(r), \mathcal{L}^{k}\right)$ well defined up to scalars. The zero locus of $\Theta_{F}$ is the set of all $E \in S U_{X}(r)$ such that $h^{0}(X, E \otimes$ $F) \neq 0$ (the degree of $F$ is such that $\chi(X, E \otimes F)=0$ ). It is easy to see that $(\dagger)$ is an isomorphism if and only if $\Theta_{F}$ for $F \in U_{X}^{*}(k) \operatorname{span} H^{0}\left(S U_{X}(r), \mathcal{L}^{k}\right)$.

Received by the editors February 23, 2006.

2000 Mathematics Subject Classification. Primary 14H60; Secondary 14D20.

The author was partially supported by NSF grant DMS-0300356. 
Let $M_{g}$ denote the moduli-space of connected smooth projective algebraic curves of genus $g$. In this paper, we prove the strange duality conjecture for generic curves:

Theorem 1.1. For generic $X \in M_{g}$, the sections $\Theta_{F}$ for $F \in U_{X}^{*}(k)$ span $H^{0}\left(S U_{X}(r), \mathcal{L}^{k}\right)$.

For the history of this problem as well as recent developments we refer the reader to Beauville [B2], Donagi-Tu [DT], Polishchuk [Po], and Popa [P].

1.1. The main idea. The starting point for this paper is the classical relation between the cohomology of Grassmannians $\operatorname{Gr}(r, n)$ and invariant theory of the special linear group $\mathrm{SL}(r)$ (or equivalently, $S U(r)$ ). In [Be1], this relation was further strengthened by demonstrating how triple intersections of Schubert varieties geometrically produce a basis of the space of invariants for the associated SL $(r)$ tensor product multiplicity problem. The next step is Witten's [W] relation between the (small) quantum cohomology of Grassmannians and structure coefficients in the Verlinde algebra for $S U(r)$ (these are dimensions of spaces of sections of theta bundles on moduli spaces of parabolic bundles on $\mathbb{P}^{1}$ ). This relation can be geometrized in a similar way. Theorem 1.1 of this paper is a higher genus generalization of this relation with $H^{0}\left(S U_{X}(r), \mathcal{L}^{k}\right)$ viewed as a representation theoretic object. The associated enumerative problem needs to be invented. The linearly independent sections coming from the inherent transversality in the enumerative problem will be shown to have the form $\Theta_{F}$ for $F \in U_{X}^{*}(k)$.

To help us invent the enumerative problem that should correspond to $H^{0}\left(S U_{X}(r), \mathcal{L}^{k}\right)$, we calculate the rank $M(r, k, g)$ of the latter (it is known that the rank of $H^{0}\left(S U_{X}(r), \mathcal{L}^{k}\right)$ does not vary with $\left.X \in M_{g}\right)$. Using a factorization formula, the dimension can be related to the dimensions of conformal blocks for $\mathbb{P}^{1}$. This reduction uses the Verlinde formula (Beauville and Laszlo BL, Faltings Fa2] and Kumar, Narasimhan, and Ramanathan KNR ), and the factorization formula of Tsuchiya, Ueno, and Yamada TUY]. By a theorem of Witten (cf. W], A]) there is a relation between conformal blocks for $\mathbb{P}^{1}$ and the (small) quantum cohomology of Grassmannians. Putting all these together, one finds a formula (see Section 8.4 for some examples):

$$
M(r, k, g)=\sum^{\prime}\left\langle\omega_{I^{1}}, \ldots, \omega_{I^{g}}, \omega_{\left(I^{1}\right)^{\prime}}, \ldots, \omega_{\left(I^{g}\right)^{\prime}}\right\rangle_{0,-k(g-1)}
$$

where the sum is over all sequences of subsets $\left(I^{1}, \ldots, I^{g}\right)$ of $[r+k]=\{1,2, \ldots, r+k\}$ with $r$ elements, each of which contains 1 , and where the Gromov-Witten invariants are "twisted" (see Section 8.2 for the definition and Section A.1 for the definition of the classes $\left.\omega_{I}\right)$.

The simultaneous appearance of Schubert cohomology classes $\omega_{I}$ and their duals $\omega_{I^{\prime}}$ in equation ( $\ddagger$ ) leads one to suspect the role of the diagonal in a product of Grassmannians $\operatorname{Gr}(r, n) \times \operatorname{Gr}(r, n)$. However, the restricted nature of the sum suggests a piece in a partial degeneration of the diagonal $\left(\Delta_{+}\right.$in Section 5D).

Using the insight given to us by equation ( $\ddagger)$, we introduce an enumerative problem that corresponds to $M(r, k, g)$ (see Section 4 ).

After this work was completed, I received a preprint from Takeshi Abe $\mathrm{Ab}$ in which he proves the strange duality conjecture for generic curves, for $r=2$, arbitrary $k$. In these cases he proves the stronger version of strange duality (generalized degrees) formulated in Donagi and Tu DT. 


\section{Determinant of the COHOMology}

Let $\mathcal{L}$ be a line bundle on a (possibly non-complete) variety $Z$ and let $s_{1}, \ldots, s_{m}$ be global sections of $\mathcal{L}$. One way of proving that $s_{1}, \ldots, s_{m}$ are linearly independent is to find points $z_{1}, \ldots, z_{m}$ in $Z$ so that the determinant of the matrix $\left(s_{i}\left(z_{j}\right)\right)$, $i=1, \ldots, m$ and $j=1, \ldots, m$, is non-zero. To make sense of this determinant, note that $s_{i}\left(z_{j}\right) \in \mathcal{L}_{z_{j}}$ and hence the determinant is an element of $\bigoplus_{j=1}^{m} \mathcal{L}_{z_{j}}$. We view $z_{j}$ as witnesses to the linear independence of the $s_{i}$.

In this section we formulate the notion of an " $(m, r, k)$-frame". Intuitively an $(m, r, k)$-frame $\left(E_{1}, \ldots, E_{m} ; F_{1}, \ldots, F_{m}\right)$ on a possibly singular irreducible curve $C$ of arithmetic genus $g$ is a tuple $\left(E_{1}, \ldots, E_{m} ; F_{1}, \ldots, F_{m}\right)$ where the $E_{j}$ are vector bundles on $C$ of rank $r$, degree 0 , and with isomorphic determinant line bundles; $F_{i}$ are vector bundles on $C$ of rank $k$ and degree $k(g-1)$, so that assuming $\operatorname{det}\left(E_{i}\right) \stackrel{\sim}{\rightarrow} \mathcal{O}$ (otherwise we will have to twist by a line bundle of degree 0 ), $E_{j} \in S U_{X}(r)$ 's are witnesses to the linear independence of the sections $\Theta_{F_{i}}$.

2.1. Determinant of cohomology and basic operations. Let $\pi: X \rightarrow S$ be a relative curve (that is, it is flat, the fibers are proper of dimension 1 , and $\left.\pi_{*}\left(\mathcal{O}_{X}\right)=\mathcal{O}_{S}\right)$. If $F$ is a coherent sheaf on $X$ which is flat over $S$, we form the determinant of its cohomology $\mathcal{D}(F)$ which is a line bundle on $S$. Formally, the fiber of $\mathcal{D}(F)$ at a point $s \in S$ is the one dimensional vector space

$$
\operatorname{det} H^{0}\left(X_{s}, F_{s}\right)^{*} \otimes \operatorname{det} H^{1}\left(X_{s}, F_{s}\right) .
$$

More precisely, the push forward in the bounded derived category $R \pi_{*}(F)$ is represented locally by a complex $\mathcal{F}_{0} \rightarrow \mathcal{F}_{1}$ of vector bundles (unique up to quasiisomorphism). Here $\mathcal{D}(F)$ is defined to be $\operatorname{det} \mathcal{F}_{0}^{*} \otimes \operatorname{det} \mathcal{F}_{1}$ (where $\operatorname{det} \mathcal{F}$ denotes the top exterior power of the vector bundle $\mathcal{F}$ ). This local definition globalizes.

Suppose that $\forall s \in S, \chi\left(F_{s}\right)=0$. Locally on $S$, there exists a complex $\mathcal{F}_{0} \stackrel{\psi}{\rightarrow} \mathcal{F}_{1}$ of vector bundles representing $R \pi_{*}(F)$ with $\operatorname{rk}\left(\mathcal{F}_{0}\right)=\operatorname{rk}\left(\mathcal{F}_{1}\right)$. Taking the top exterior product of $\psi$, we find a canonical section $\sigma(F) \in \mathcal{D}(F)$. It is easy to see that for $s \in S, \sigma\left(F_{s}\right)=0$ if and only if $h^{0}\left(X_{s}, F_{s}\right) \neq 0$.

The determinant of cohomology and its canonical section satisfy some compatibility properties:

(1) If $\alpha: 0 \rightarrow F_{1} \rightarrow F_{2} \rightarrow F_{3} \rightarrow 0$ is an exact sequence of $S$-flat coherent sheaves on $X$, then there is an induced isomorphism

$$
\mathcal{D}(\alpha): \mathcal{D}\left(F_{2}\right) \stackrel{\sim}{\rightarrow} \mathcal{D}\left(F_{1}\right) \otimes \mathcal{D}\left(F_{3}\right) .
$$

(2) If, in (1), the relative Euler characteristics of $F_{1}, F_{2}$, and $F_{3}$ are each zero, then

$$
\mathcal{D}(\alpha)\left(\sigma\left(F_{2}\right)\right)=\sigma\left(F_{1}\right) \otimes \sigma\left(F_{3}\right) .
$$

In [Fa1], G. Faltings makes the following definition.

Definition 2.1. Let $I$ and $J$ be $S$-flat coherent sheaves on $X$. We say $I$ and $J$ coincide generically in $K$-theory if the following holds: For all $s \in S$ such that $\operatorname{depth}\left(\mathcal{O}_{S, s}\right)=0$, the difference of $I$ and $J$ in the $K$-theory of coherent $\mathcal{O}_{S, s}$ flat sheaves on $X \times{ }_{S} \operatorname{Spec}\left(\mathcal{O}_{S, s}\right)$ is represented by a finite complex of sheaves, such that the support of its cohomology is finite over $\operatorname{Spec} \mathcal{O}_{S, s}$.

If, for example, $X$ and $S$ are reduced and irreducible and $I$ and $J$ are $S$-flat and have the same generic rank (that is, rank at the generic point of $X$ ), then they coincide generically in $K$-theory. This case will be sufficient for this paper. 
Faltings then proves (Theorem I.1 of [Fa1]) that if $I$ and $J$ are two $S$-flat coherent sheaves on $X$ of the same relative Euler characteristic and which coincide generically in $K$-theory and if $E, E^{\prime}$ are vector bundles on $X$ of the same rank with a given isomorphism $\psi: \operatorname{det}(E) \rightarrow \operatorname{det}\left(E^{\prime}\right)$, there is a natural isomorphism

$$
\Phi_{E, E^{\prime}}(\psi): \mathcal{D}(E \otimes I) \otimes \mathcal{D}\left(E^{\prime} \otimes J\right) \stackrel{\sim}{\rightarrow} \mathcal{D}\left(E^{\prime} \otimes I\right) \otimes \mathcal{D}(E \otimes J)
$$

with various functorial and compatibility properties (compatible exact sequences in $\left.\left(E, E^{\prime}\right)\right)$. We will always assume that $I$ and $J$ are in addition also of the same Euler characteristic. This ensures that $\Phi_{E, E^{\prime}}(\psi)$ does not depend upon the choice of the isomorphism $\psi: \operatorname{det}(E) \stackrel{\sim}{\rightarrow} \operatorname{det}\left(E^{\prime}\right)$. We will therefore drop the dependence on $\psi$ in the maps $\Phi_{E, E^{\prime}}$.

In the remainder of this section we will recall the construction of $\Phi_{E, E^{\prime}}$ and note its basic properties.

Consider the special case when there exist line bundles $L_{1}, \ldots, L_{k}$ and filtrations

$$
\begin{aligned}
& E=E_{1} \supset E_{2} \supset \cdots \supset E_{r} \supset 0=E_{r+1}, \\
& E^{\prime}=E_{1}^{\prime} \supset E_{2}^{\prime} \supset \cdots \supset E_{r}^{\prime} \supset 0=E_{r+1}^{\prime}
\end{aligned}
$$

and (given) isomorphisms $E_{j} / E_{j+1} \rightarrow L_{j}$ and $E_{j}^{\prime} / E_{j+1}^{\prime} \rightarrow L_{j}$ for $j=1, \ldots, r$. Such a situation can be arranged after a suitable flat base change of $S$ (for details see Fa1]). If $S=\operatorname{Spec}(\mathbb{C})$, no base changes are needed! In such a set up there are induced isomorphisms

$$
\begin{array}{ll}
A: \mathcal{D}(E \otimes I) \rightarrow \prod_{j=1}^{r} \mathcal{D}\left(L_{j} \otimes I\right), & B: \mathcal{D}\left(E^{\prime} \otimes J\right) \rightarrow \prod_{j=1}^{r} \mathcal{D}\left(L_{j} \otimes J\right), \\
C: \mathcal{D}\left(E^{\prime} \otimes I\right) \rightarrow \prod_{j=1}^{r} \mathcal{D}\left(L_{j} \otimes I\right), & D: \mathcal{D}(E \otimes J) \rightarrow \prod_{j=1}^{r} \mathcal{D}\left(L_{j} \otimes J\right) .
\end{array}
$$

The isomorphism $\Phi_{E, E^{\prime}}$ in "this good coordinate system" is just the identity map. That is, the diagram below commutes (where the bottom arrow is identity):

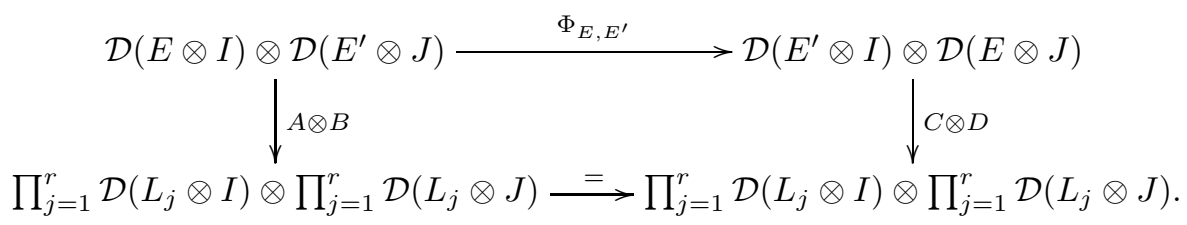

Faltings shows that $\Phi_{E, E^{\prime}}$ is independent of the good coordinates (by this we mean the choice of filtrations of $E$ and $E^{\prime}$ ). Together with [KM], Proposition 1(ii), it is easy to see that $\Phi_{E, E^{\prime}}$ is compatible with exact sequences in $(I, J)$. That is, if

$$
0 \rightarrow I_{1} \rightarrow I \rightarrow I_{2} \rightarrow 0,0 \rightarrow J_{1} \rightarrow J \rightarrow J_{2} \rightarrow 0
$$

are exact sequences of $S$-flat coherent sheaves such that $I_{a}, J_{a}$ have the same relative Euler characteristics for $a=1,2$ and coincide generically in $K$-theory, then the following diagram commutes ( $E$ and $E^{\prime}$ are as above):

$$
\begin{array}{cc}
\mathcal{D}(E \otimes I) \otimes \mathcal{D}\left(E^{\prime} \otimes J\right) \stackrel{\Phi_{E, E^{\prime}}}{\longrightarrow} \mathcal{D}\left(E^{\prime} \otimes I\right) \otimes \mathcal{D}(E \otimes J) \\
\downarrow & \downarrow \\
D\left(E \otimes I_{1}\right) \otimes \mathcal{D}\left(E^{\prime} \otimes J_{1}\right) \otimes \stackrel{\Phi_{E, E^{\prime}}}{\longrightarrow} D\left(E^{\prime} \otimes I_{1}\right) \otimes \mathcal{D}\left(E \otimes J_{1}\right) \otimes \\
D\left(E \otimes I_{2}\right) \otimes \mathcal{D}\left(E^{\prime} \otimes J_{2}\right) & D\left(E^{\prime} \otimes I_{2}\right) \otimes \mathcal{D}\left(E \otimes J_{2}\right)
\end{array}
$$


where the bottom $\Phi_{E, E^{\prime}}$ is the tensor product of $\Phi_{E, E^{\prime}}$ acting on the two levels. Faltings does not list this property, and we learned it from the proof of Lemma 1 in $[\mathrm{E}]$.

The second observation, which is obvious in good coordinates, is that if $I=J$, then the isomorphism $\Phi_{E, E^{\prime}}$ is just the permutation of the two factors (if $L$ is a one dimensional vector space with $\ell_{1}, \ell_{2} \in L$, then $\left.\ell_{1} \otimes \ell_{2}=\ell_{2} \otimes \ell_{1} \in L \otimes L\right)$.

The third observation is that if $\left(E_{1}, \ldots, E_{m} ; F_{1}, \ldots, F_{m}\right)$ are vector bundles on $X$ such that $E_{1}, \ldots, E_{m}$ are vector bundles with isomorphic determinants and each of rank $r, F_{1}, \ldots, F_{m}$ are vector bundles on $X$ of the same relative degree and rank and if $\pi \in S_{n}$ (the symmetric group on $\{1, \ldots, n\}$ ), then there are "natural" isomorphisms, compatible with compositions:

$$
\begin{aligned}
\mathcal{D}\left(E_{1} \otimes F_{1}\right) & \otimes \mathcal{D}\left(E_{2} \otimes F_{2}\right) \otimes \ldots \otimes \mathcal{D}\left(E_{m} \otimes F_{m}\right) \\
& \stackrel{A_{\pi}}{\rightarrow} \mathcal{D}\left(E_{\pi(1)} \otimes F_{1}\right) \otimes \mathcal{D}\left(E_{\pi(2)} \otimes F_{2}\right) \otimes \ldots \otimes \mathcal{D}\left(E_{\pi(m)} \otimes F_{m}\right) .
\end{aligned}
$$

To see this, there are such maps for transpositions $\pi$ viz. $\Phi_{E_{i}, E_{j}}$. Writing any permutation as a composition of transpositions, we will obtain maps for all $\pi$. The independence from the choice of the representation of $\pi$ as a composition of permutations (as well as compatibility properties) follows from the following: "in good coordinates for $E_{i}$ " all these maps are the identity.

2.2. Frames of vector bundles on curves. Let $C$ be a reduced irreducible projective algebraic curve of arithmetic genus $g$ with only ordinary double points for singularities. Consider a tuple $\left(E_{1}, \ldots, E_{m} ; F_{1}, \ldots, F_{m}\right)$ where

(P1) $E_{1}, \ldots, E_{m}$ are vector bundles on $C$ each of degree 0 , rank $r$, and such that for $i, j \in\{1, \ldots, m\}, \operatorname{det}\left(E_{i}\right)$ is isomorphic to $\operatorname{det}\left(E_{j}\right)$,

(P2) $F_{1}, \ldots, F_{m}$ are vector bundles on $C$, each of rank $k$ and degree $k(g-1)$.

Notice that $\chi\left(C, E_{i} \otimes F_{j}\right)=0$ and hence we have canonical elements $\sigma\left(E_{i} \otimes F_{j}\right) \in$ $\mathcal{D}\left(E_{i} \otimes F_{j}\right)$ (a one dimensional vector space).

By the third observation in Section 2.1, the one dimensional vector spaces for various $\pi \in S_{n}$ (the symmetric group)

$$
\mathcal{D}\left(E_{\pi(1)} \otimes F_{1}\right) \otimes \mathcal{D}\left(E_{\pi(2)} \otimes F_{2}\right) \otimes \ldots \otimes \mathcal{D}\left(E_{\pi(m)} \otimes F_{m}\right)
$$

are all canonically identified. Consider the $m \times m$ matrix $\left(\sigma\left(E_{i} \otimes F_{j}\right)\right), i=1, \ldots, m$, $j=1, \ldots, m$. Because of the remark above it makes sense to speak about the nonzeroness of the determinant of this matrix.

Definition 2.2. A tuple $\left(E_{1}, \ldots, E_{m} ; F_{1}, \ldots, F_{m}\right)$ satisfying properties $(\mathrm{P} 1)$ and (P2) is said to be an $(m, r, k)$-frame on $C$ if the determinant of the $m \times m$ matrix $\left(\sigma\left(E_{i} \otimes F_{j}\right)\right), i=1, \ldots, m, j=1, \ldots, m$, is non-zero.

We will give a geometric interpretation of the definition of a frame. Let $\left(E_{1}, \ldots\right.$, $\left.E_{m} ; F_{1}, \ldots, F_{m}\right)$ be a tuple as above. Fix a vector bundle $E_{0}$ of the same rank as $E_{i}$ 's whose determinant is isomorphic to that of $E_{i}$ (for example one of the $E_{i}$ ). Let $F_{0}$ be a vector bundle of the same degree and rank as the $F_{j}$ 's. Choose nonzero elements $s_{j} \in \mathcal{D}\left(E_{0} \otimes F_{j}\right)$ and $t \in \mathcal{D}\left(E_{0} \otimes F_{0}\right)$. These choices do not involve $E_{1}, \ldots, E_{m}$. We have canonical isomorphisms

$$
\Phi_{E, E_{0}}: \mathcal{D}\left(E \otimes F_{i}\right) \otimes \mathcal{D}\left(E_{0} \otimes F_{0}\right) \stackrel{\sim}{\rightarrow} \mathcal{D}\left(E_{0} \otimes F_{i}\right) \otimes \mathcal{D}\left(E \otimes F_{0}\right) .
$$

The choice of $t, s_{0}, \ldots, s_{m}$ gives a morphism $\lambda_{j}: \mathcal{D}\left(E \otimes F_{j}\right) \rightarrow \mathcal{D}\left(E \otimes F_{0}\right)$. 
Therefore one obtains $m$ tuples of complex numbers well defined up to non-zero scalars $\Theta(E)=\left[\lambda_{1}\left(\sigma\left(E \otimes F_{1}\right)\right), \ldots, \lambda_{m}\left(\sigma\left(E \otimes F_{m}\right)\right)\right]$. But $\Theta(E)$ (up to non-zero scalars) does depend upon the choice of $\left(E_{0}, F_{0}, t, s_{1}, \ldots, s_{m}\right)$. For example, if we change $s_{i}$, then the entry $i$ of $\Theta(E)$ gets scaled.

It is easy to verify that $\left(E_{1}, \ldots, E_{m} ; F_{1}, \ldots, F_{m}\right)$ is an $(m, r, k)$-frame if and only if $\Theta\left(E_{1}\right), \ldots, \Theta\left(E_{m}\right)$ are linearly independent. The main properties of $(m, r, k)$ frames are

Proposition 2.3. Let $X \in M_{g}$ and let $\left(E_{1}, \ldots, E_{m} ; F_{1}, \ldots, F_{m}\right)$ be an $(m, r, k)$ frame on $X$ with $\operatorname{det}\left(E_{i}\right) \stackrel{\sim}{\rightarrow} \mathcal{O}_{X}$. Then $\Theta_{F_{j}} \in H^{0}\left(S U_{X}(r), \mathcal{L}^{k}\right), j=1, \ldots, m$, are linearly independent. If $m \geq \operatorname{rk} H^{0}\left(S U_{X}(r), \mathcal{L}^{k}\right)$, these sections give a basis of $H^{0}\left(S U_{X}(r), \mathcal{L}^{k}\right)$ (and hence $m=\operatorname{rk} H^{0}\left(S U_{X}(r), \mathcal{L}^{k}\right)$.)

Proof. From the determinantal condition in the definition of the frame, we see that for each $i$ there exists a $j$ (similarly for each $j$ there is an $i$ ) so that $\sigma\left(E_{i} \otimes F_{j}\right) \neq 0$. Therefore by Lemma A.1 $E_{i}$ and $F_{j}$ are semi-stable vector bundles on $X$ for all $i$ and $j$. For the linear independence we can replace $S U_{X}(r)$ by a space $Z$ that carries a universal family of semi-stable vector bundles of trivial determinant and rank $r$ on $X$. The same determinantal condition now implies the linear independence of $\Theta_{F_{j}}$.

Lemma 2.4. Suppose that an $(m, r, k)$-frame $\left(E_{1}, \ldots, E_{m} ; F_{1}, \ldots, F_{m}\right)$ exists on a reduced irreducible projective curve $C$ with at most double point singularities. Then there exists such an $(m, r, k)$-frame on $C$ with $\operatorname{det}\left(E_{i}\right)$ isomorphic to $\mathcal{O}_{C}$.

Proof. The group of line bundles of degree 0 on $C$ is a divisible group. Suppose that $L^{r} \stackrel{\sim}{\rightarrow} \operatorname{det}\left(E_{i}\right)$ (recall that we are assuming that $E_{i}$ is of degree 0 ). Then we consider the modified frame

$$
\left(E_{1} \otimes L^{-1}, \ldots, E_{m} \otimes L^{-1} ; F_{1} \otimes L, \ldots, F_{m} \otimes L\right) .
$$

Lemma 2.5. Consider a projective flat family $X \rightarrow S$ of curves. Let $s_{0} \in S$ be such that $X_{s_{0}}$ is a reduced irreducible curve with at most ordinary double point singularities. Suppose that an $(m, r, k)$-frame exists on $X_{s_{0}}$. Then there is an open subset $U \subseteq S$ containing $s_{0}$ such that $X_{s}$ has an $(m, r, k)$-frame for each $s \in U$.

Proof. Let $\left(E_{1}, \ldots, E_{m} ; F_{1}, \ldots, F_{m}\right)$ be an $(m, r, k)$-frame on $X_{s_{0}}$. Using Lemma2.4. assume that $\operatorname{det}\left(E_{i}\right) \stackrel{\sim}{\rightarrow} \mathcal{O}_{X_{s_{0}}}$ for $i=1, \ldots, m$.

Using Lemma A.2, find an étale base change $S^{\prime} \rightarrow S$ so that the image contains $s_{0}$ and $\left(E_{1} \ldots, E_{m} ; F_{1}, \ldots, F_{m}\right)$ lift to vector bundles on $X_{S^{\prime}}$ satisfying properties (a) and (b) of the frame. The non-vanishing of the determinant is an open condition and this concludes the proof.

\section{Line BUNDLES AND VECTOR BUNDLES ON RATIONAL NODAL CURVES}

Let us fix a rational nodal curve $C$ of arithmetic genus $g$ together with a normalization $\mathbb{P}^{1} \stackrel{f}{\rightarrow} C$. Let $r_{1}, \ldots, r_{g}$ be the nodes of $C$ and let $f^{-1}\left(r_{j}\right)=\left\{p_{j}, q_{j}\right\}$ for $j=1, \ldots, g$. This notation will be fixed throughout the paper.

Let $L$ be a line bundle of degree 0 on $C$ (that is, the degree of $f^{*} L$ is zero). It is clear that $f^{*} L$ is trivial; that is, there is a isomorphism unique up to scalars 
$A: \mathcal{O}_{\mathbb{P}^{1}} \rightarrow f^{*} L$. The canonical map $f^{*}(L)_{p_{j}} \rightarrow f^{*}(L)_{q_{j}}$ gives us a well defined scalar $c_{j}$ which makes the following diagram commute:

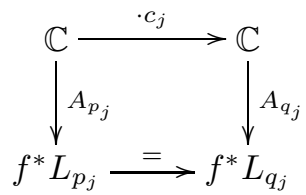

We therefore obtain a well defined morphism of groups

$$
\{\text { line bundles of degree } 0 \text { on } \mathrm{C}\} \rightarrow\left(\mathbb{C}^{*}\right)^{g}, L \mapsto\left(c_{1}, \ldots, c_{g}\right) \text {. }
$$

It is easily checked that this is a group isomorphism.

\section{1. $S$-bundles on rational nodal curves.}

Definition 3.1. An $S$-bundle on $C$ is a pair $(V, \tau)$ where $V$ is a vector bundle on $\mathbb{P}^{1}$ together with a $g$-tuple $\tau=\left(\tau_{1}, \ldots, \tau_{g}\right)$ where $\tau_{j}: V_{p_{j}} \rightarrow V_{q_{j}}$ for $j=1, \ldots, g$ are morphisms of vector spaces.

Consider the surjective morphism $f_{*} V \rightarrow \bigoplus_{j} V_{q_{j}} \mid r_{j}\left(V_{q_{j}} \mid r_{j}\right.$ is the skyscraper sheaf supported at $r_{j}$ with fiber $V_{q_{j}}$ ) corresponding to the map $V_{p_{j}} \oplus V_{q_{j}} \rightarrow V_{q_{j}}$ given by $-\tau_{j}$ on the first factor and the identity on the second. Let $\tilde{V}$ be the kernel. It is a coherent sheaf on $C$ and will be called the coherent sheaf underlying $(V, \tau)$. It is easy to see that if the $\tau_{j}$ are isomorphisms for $j=1, \ldots, g$, then $\tilde{V}$ is the vector bundle on $C$ obtained by gluing $V_{p_{j}}$ and $V_{q_{j}}$ via the maps $\tau_{j}$, with a given isomorphism $f^{*} \tilde{V} \stackrel{\sim}{\rightarrow} V$.

Let $(V, \tau)$ and $(Q, \theta)$ be $S$-bundles as above. A morphism $(V, \tau) \rightarrow(Q, \theta)$ is a morphism $\Gamma: V \rightarrow Q$ on $\mathbb{P}^{1}$ such that for $j=1, \ldots, g$ the diagram

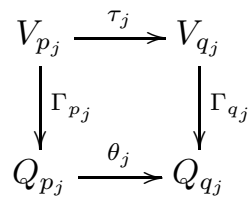

commutes. The (vector) space of such homomorphisms is denoted by $\underline{\operatorname{Hom}}((V, \tau)$, $(Q, \theta))$.

Let $\left(V, \tau^{(1)}\right)$ and $\left(V, \tau^{(2)}\right)$ be $S$-bundles on $C$ with $V=\mathcal{O}_{\mathbb{P}^{1}}^{\oplus r}$ such that $\tau_{j}^{(i)}$ are isomorphisms for $i=1,2, j=1, \ldots, g$. These give rise to coherent sheaves $\tilde{V}_{1}$ and $\tilde{V}_{2}$ on $C$ which are locally free sheaves. We view $\tau_{j}^{(1)}$ and $\tau_{j}^{(2)}$ as $r \times r$ matrices using the identification $V \stackrel{\sim}{\rightarrow} \mathcal{O}_{\mathbb{P}^{1}}^{\oplus}$. The following lemma is immediate:

Lemma 3.2. As line bundles on $C, \operatorname{det}\left(\tilde{V}_{1}\right)$ and $\operatorname{det}\left(\tilde{V}_{2}\right)$ are isomorphic if and only if $\operatorname{det}\left(\tau_{j}^{(1)}\right)=\operatorname{det}\left(\tau_{j}^{(2)}\right) \in \mathbb{C}^{*}$ for $j=1, \ldots, g$.

\section{Outline of the argument}

Lemmas 2.5, 2.4 and Proposition 2.3 give a strategy for proving the strange duality conjecture for generic $X \in M_{g}$. 
Initial strategy. Construct an $(m, r, k)$-frame on the rational nodal curve $C$ of arithmetic genus $g$ with $m \geq M(r, k, g)(M(r, k, g)$ was defined in the introduction).

Here we use the fact that there exists a family of projective curves degenerating into $C$, such that the general member of this family is a curve in $M_{g}$.

An $(m, r, k)$-frame on $C$ will be constructed by working on the normalization $\mathbb{P}^{1}$ of $C$. We will first recall some relevant definitions and properties of "evenly split" bundles on $\mathbb{P}^{1}$.

4.1. Evenly split bundles on $\mathbb{P}^{1}$. A vector bundle $T$ on $\mathbb{P}^{1}$ is said to be evenly split (ES) if $W=\bigoplus_{i=1}^{n} \mathcal{O}_{\mathbb{P}^{1}}\left(a_{i}\right)$ with $\left|a_{i}-a_{j}\right| \leq 1$ for $0<i<j \leq n$.

Let $D$ and $n$ be integers with $n>0$. It is easy to show there is a unique, up to isomorphism, ES-bundle of degree $-D$ and rank $n$ on $\mathbb{P}^{1}$ (the negative sign is introduced for conformity with notation used in quantum cohomology).

Let $T$ be a vector bundle on $\mathbb{P}^{1}$ of degree $-D$ and rank $n$. Define $\operatorname{Gr}(d, r, T)$ to be the moduli space of subbundles of $T$ which are of degree $-d$ and rank $r$. This can be obtained as an open subset of the quot scheme of quotients of $T$ of degree $d-D$ and rank $n-r$. In the notation of Pot, we are looking at the open subset of Hilb ${ }^{n-r, d-D}(T)$ formed by points where the quotient is locally free. The proof of the following standard result may be found in [Be2].

Proposition 4.1. Let $T$ be an ES bundle of degree $-D$ and rank $n$. Then $\operatorname{Gr}(d, r, T)$ is smooth and irreducible of dimension $r(n-r)+d n-D r$. Moreover, the subset of $\operatorname{Gr}(d, r, T)$ formed by ES-subbundles $V \subseteq T$ such that $T / V$ is also $E S$ is open and dense in $\operatorname{Gr}(d, r, T)$.

4.2. The enumerative problem and the resulting frame on $C$. Let $T$ be an ES-vector bundle of rank $n=r+k$ and degree $k(g-1)$ on $\mathbb{P}^{1}$. Fix a generic tuple

$$
\left(\gamma_{1}, \ldots, \gamma_{g}\right) \in \prod_{j=1}^{g} \operatorname{Hom}_{n-1}\left(T_{p_{j}}, T_{q_{j}}\right)
$$

where $\operatorname{Hom}_{n-1}\left(T_{p_{j}}, T_{q_{j}}\right)$ denotes the set of maps of vector spaces $T_{p_{j}} \rightarrow T_{q_{j}}$ of rank $n-1$ (therefore the kernel of $\gamma_{j}$ is one dimensional.)

The enumerative problem is the following: Count the number of "singular Ssubbundles" $(V, \tau)$ of $(T, \gamma)$ so that $\operatorname{deg}(V)=0$ and $\operatorname{rk}(V)=r$. More precisely, we want to count subbundles $V$ of $T$ of degree 0 and rank $r$ so that for $j=1, \ldots, g$,

- $\gamma_{j}\left(V_{p_{j}}\right) \subset V_{q_{j}}$,

- the induced map $\tau_{j}: V_{p_{j}} \rightarrow V_{q_{j}}$ is singular.

In Proposition 6.1, we will show that there are only finitely many such S-subbundles and that the natural parameter scheme of such objects is reduced. The number $m$ of these will be shown to be $\geq M(r, k, g)$ (Proposition 6.2).

Denote them by $V^{(a)}$ for $a=1, \ldots, m$. Let $Q^{(a)}=T / V^{(a)}$ for $a=1, \ldots, m$. Both $V^{(a)}$ and $Q^{(a)}$ have natural S-bundle structures. We therefore obtain S-bundles $\left(V^{(a)}, \tau^{(a)}\right)$ and $\left(Q^{(a)}, \theta^{(a)}\right)$. We will show that the gluing maps $\theta_{j}^{(a)}$ for $Q^{(a)}$ are nonsingular (Lemma 6.4(ii)). Hence the coherent sheaf underlying $\left(Q^{(a)}, \theta^{(a)}\right)$ is a vector bundle $\tilde{Q}^{(a)}$ of degree $k(g-1)$.

We perturb the maps $\tau^{(a)}$ (and leave maps $\theta^{(a)}$ unchanged) so that they become non-singular and such that the corresponding vector bundles (on $C$ ) $\tilde{V}^{(a)}$ have isomorphic determinant line bundles for $a=1, \ldots, m$. This perturbation is possible 
because we are starting from singular maps $\tau_{j}^{(a)}$. Finally we will show, using the transversality in the enumerative problem, that the tuple

$$
\left(\left(\tilde{V}^{(1)}\right)^{*}, \ldots,\left(\tilde{V}^{(m)}\right)^{*} ; \tilde{Q}^{(1)}, \ldots, \tilde{Q}^{(m)}\right)
$$

is an $(m, r, k)$-frame on the rational nodal curve $C$, using the fact that in the limit, an appropriate matrix is diagonal with non-zero entries on the diagonal. This will conclude the proof.

\section{Reformulation in terms of the diagonal}

The enumerative problem given in Section 4.2 is a counting problem of the number of "singular" subbundles of a bundle on $C$ with singular gluing maps. However recall that if $\tau_{j}$ were non-singular, then the set of subbundles of the induced bundle $\tilde{T}$ (on $C$ ) of degree 0 and rank $r$ can be described as follows: Consider the natural map

$$
\pi: \operatorname{Gr}(0, r, T) \rightarrow \prod_{j=1}^{g}\left(\operatorname{Gr}\left(r, T_{p_{j}}\right) \times \operatorname{Gr}\left(r, T_{q_{j}}\right)\right) .
$$

Since there is a given isomorphism $\gamma_{j}: T_{p_{j}} \rightarrow T_{q_{j}}$, we can define a "diagonal"

$$
\Delta_{j}=\left\{(A, B) \in \operatorname{Gr}\left(r, T_{p_{j}}\right) \times \operatorname{Gr}\left(r, T_{q_{j}}\right) \mid \gamma_{j}(A)=B\right\} .
$$

The set of subbundles of $\tilde{T}$ of degree 0 and rank $r$ is just $\pi^{-1} \prod_{j=1}^{g} \Delta_{j}$.

In Section [6] we show that the enumerative problem in Section 4 has a similar description, but we need to replace $\Delta_{j}$ by a singular "diagonal". To obtain transversality in the enumerative problem and to make use of it, we will need to study tangent spaces as well.

5.1. Degeneration of the diagonal. Let $W$ be a vector space of rank $n$ and $0<r<n$ an integer. We will consider partial degenerations of the diagonal $\Delta=\{(A, B) \in \operatorname{Gr}(r, W) \times \operatorname{Gr}(r, W) \mid A=B\}$.

If $\Phi \in \operatorname{End}(W)$, then define

$$
\Delta_{\Phi}=\{(A, B) \in \operatorname{Gr}(r, W) \times \operatorname{Gr}(r, W) \mid \Phi(A) \subseteq B\} .
$$

We therefore obtain a subscheme

$$
\tilde{\Delta} \subseteq \operatorname{Gr}(r, W) \times \operatorname{Gr}(r, W) \times \operatorname{End}(W)
$$

such that the fiber of the projection $T: \tilde{\Delta} \rightarrow \operatorname{End}(W)$ over $\Phi$ is $\Delta_{\Phi}$. Clearly $T$ is flat over $\operatorname{Aut}(W)$. The map $\tilde{\Delta} \rightarrow \operatorname{Gr}(r, W) \times \operatorname{Gr}(r, W)$ given by $(A, B, \Phi) \mapsto(A, B)$ is Zariski locally, a trivial fiber bundle with smooth fibers. Therefore $\tilde{\Delta}$ is smooth.

We claim that $T$ is flat over endomorphisms of rank $\geq n-1$. It suffices to show that if $\Phi$ is singular with kernel $L$ of rank 1 , then $\Delta_{\Phi}$ is equidimensional of dimension $r(n-r)$. If $(A, B) \in \Delta_{\Phi}$, then either $L \subset A$ or $B \subset \operatorname{im}(\Phi)$. These two (irreducible) components each have the correct dimension (by a small calculation, for example, the second dimension is $\operatorname{dim}(\operatorname{Gr}(r, n-1))+\operatorname{dim}(\operatorname{Gr}(r, r+1)))$.

Let $L=\mathbb{C} \ell \subseteq W$ and $K \subseteq W$ be subspaces of ranks 1 and $n-1$, respectively, so that the natural map $L \oplus K \rightarrow W$ is an isomorphism. Let $\beta: W \rightarrow W$ be the corresponding projection to $K$. Here $\Delta_{\beta}$ is a union $\Delta_{+} \cup \Delta_{-}$where

$$
\begin{aligned}
& \Delta_{+}=\{(A, B) \in \operatorname{Gr}(r, W) \times \operatorname{Gr}(r, W) \mid \beta(A) \subseteq B, L \subseteq A\}, \\
& \Delta_{-}=\{(A, B) \in \operatorname{Gr}(r, W) \times \operatorname{Gr}(r, W) \mid \beta(A) \subseteq B, B \subseteq K\} .
\end{aligned}
$$


Let $K=\operatorname{Span}\left\{k_{2}, \ldots, k_{n}\right\}$. Define complete flags on $W$ :

$$
\begin{aligned}
& F_{\bullet}: 0 \subsetneq F_{1} \subsetneq F_{2} \subsetneq \cdots \subsetneq F_{n}=W, \\
& F_{\bullet}^{\prime}: 0 \subsetneq F_{1}^{\prime} \subsetneq F_{2}^{\prime} \subsetneq \cdots \subsetneq F_{n}^{\prime}=W,
\end{aligned}
$$

as follows: $F_{1}=L, F_{i}=L \oplus \operatorname{Span}\left\{k_{2}, \ldots, k_{i}\right\}$ for $i=2, \ldots, n$, and $F_{i}^{\prime}=$ $\operatorname{Span}\left\{k_{n}, \ldots, k_{n-i+1}\right\}$ for $i=1, \ldots, n-1$ and $F_{n}^{\prime}=W$. Define $\phi_{a}(t): W \rightarrow W$ for $a=1, \ldots, n$ as follows:

- $\phi_{1}(t)$ is multiplication by $t$ on $L$ and identity on $K$,

- for $a=2, \ldots, n, \phi_{a}(t)$ is multiplication by $t$ on $F_{a}$ and $\phi_{a}(t)\left(k_{i}\right)=k_{i}$ for $i>a$.

For a subset $I$ of $[n]$ of cardinality $r$, let $I^{\prime}=\{n+1-i, i \in I\}$ (see Section A.1). It is well known that the cycle class (in cohomology) of the diagonal $\Delta \subseteq \operatorname{Gr}(r, W) \times$ $\operatorname{Gr}(r, W)$ is the sum of cycle classes of $\Omega_{I}\left(F_{\bullet}\right) \times \Omega_{I^{\prime}}\left(F_{\bullet}^{\prime}\right)$, each with multiplicity one. There is actually a geometric degeneration of $\Delta$ into this union of products of Schubert varieties $\left[\mathrm{BP}\right.$. The degenerations below of $\Delta, \Delta_{+}$, and $\Delta_{-}$are built in a similar manner.

Proposition 5.1. Consider the following limits in the Hilbert scheme of $\operatorname{Gr}(r, W) \times$ $\operatorname{Gr}(r, W)$ :

$$
\begin{gathered}
\delta=\lim _{t_{n} \rightarrow 0}\left(1 \times \phi_{n}\left(t_{n}\right)\right) \lim _{t_{n-1} \rightarrow 0}\left(1 \times \phi_{n-1}\left(t_{n-1}\right)\right) \ldots \lim _{t_{1} \rightarrow 0}\left(1 \times \phi_{1}\left(t_{1}\right)\right) \Delta, \\
\delta_{+}=\lim _{t_{n} \rightarrow 0}\left(1 \times \phi_{n}\left(t_{n}\right)\right) \lim _{t_{n-1} \rightarrow 0}\left(1 \times \phi_{n-1}\left(t_{n-1}\right)\right) \ldots \lim _{t_{2} \rightarrow 0}\left(1 \times \phi_{2}\left(t_{2}\right)\right) \Delta_{+}, \\
\delta_{-}=\lim _{t_{n} \rightarrow 0}\left(1 \times \phi_{n}\left(t_{n}\right)\right) \lim _{t_{n-1} \rightarrow 0}\left(1 \times \phi_{n-1}\left(t_{n-1}\right)\right) \ldots \lim _{t_{2} \rightarrow 0}\left(1 \times \phi_{2}\left(t_{2}\right)\right) \Delta_{-} .
\end{gathered}
$$

Then, $\delta, \delta_{+}$, and $\delta_{-}$coincide set-theoretically with the schemes $\bigcup_{I} \Omega_{I}\left(F_{\bullet}\right) \times \Omega_{I^{\prime}}\left(F_{\bullet}^{\prime}\right)$, $\bigcup_{I: 1 \in I} \Omega_{I}\left(F_{\bullet}\right) \times \Omega_{I^{\prime}}\left(F_{\bullet}^{\prime}\right)$, and $\bigcup_{I: 1 \notin I} \Omega_{I}\left(F_{\bullet}\right) \times \Omega_{I^{\prime}}\left(F_{\bullet}^{\prime}\right)$, respectively. This identification is scheme-theoretic over the smooth open dense subschemes (in each) $\bigcup_{I} \Omega_{I}^{o}\left(F_{\bullet}\right) \times \Omega_{I^{\prime}}^{o}\left(F_{\bullet}^{\prime}\right), \bigcup_{I: 1 \in I} \Omega_{I}^{o}\left(F_{\bullet}\right) \times \Omega_{I^{\prime}}^{o}\left(F_{\bullet}^{\prime}\right)$, and $\bigcup_{I: 1 \notin I} \Omega_{I}^{o}\left(F_{\bullet}\right) \times \Omega_{I^{\prime}}^{o}\left(F_{\bullet}^{\prime}\right)$, respectively.

Remark 5.2. By Lemma 6 in $\left[\mathrm{BL}\right.$, the class $\left[\mathcal{O}_{\Delta}\right]$ of $\Delta$ in the $K$-theory of coherent sheaves on $\operatorname{Gr}(r, W) \times \operatorname{Gr}(r, W)$ coincides with the class of the reduced scheme $\bigcup_{I} \Omega_{I}\left(F_{\bullet}\right) \times \Omega_{I^{\prime}}\left(F_{\bullet}^{\prime}\right)$. It now follows from the above proposition (by considering Hilbert polynomials) that $\delta$ equals $\bigcup_{I} \Omega_{I}\left(F_{\bullet}\right) \times \Omega_{I^{\prime}}\left(F_{\bullet}^{\prime}\right)$ as schemes. Similar statements hold for $\delta_{+}$and $\delta_{-}$. We will however not use this refinement of Proposition 5.1

Proof. We proceed by induction on $n$. First degenerate, as before,

$$
\lim _{t_{1} \rightarrow 0}\left(1 \times \phi_{1}\left(t_{1}\right)\right) \Delta=\Delta_{+} \cup \Delta_{-} .
$$

It suffices therefore to prove the statements about $\delta_{+}$and $\delta_{-}$. The case of $\delta_{-}$is similar to that of $\delta_{+}$, so we will prove only the statements for $\delta_{+}$. For any subscheme $X$ of $\operatorname{Gr}(r-1, K) \times \operatorname{Gr}(r-1, K)$, let $\mathcal{T}(X)$ be the $\mathbb{P}^{n-r}$ bundle over $X$ given by

$$
\begin{aligned}
\mathcal{T}(X)=\left\{\left(A, B, A^{\prime}, B^{\prime}\right)\right. & \in \operatorname{Gr}(r, W) \times \operatorname{Gr}(r, W) \times X: \\
& \left.\left(A^{\prime}, B^{\prime}\right) \in X, A=A^{\prime} \oplus L, B \supset B^{\prime}\right\} .
\end{aligned}
$$

The natural map $\mathcal{T}(X) \rightarrow \operatorname{Gr}(r, W) \times \operatorname{Gr}(r, W)$ is birational onto its image. 
Clearly $\mathcal{T}(\Delta(r-1, K)) \stackrel{\sim}{\rightarrow} \Delta_{+}$, where $\Delta(r-1, K)$ is the diagonal in $\operatorname{Gr}(r-1, K) \times \operatorname{Gr}(r-1, K)$. The operator $\mathcal{T}$ can be applied to families of subschemes of $\operatorname{Gr}(r-1, K) \times \operatorname{Gr}(r-1, K)$. Now apply the operator $\mathcal{T}$ to the inductively obtained degeneration of $\Delta(r-1, K)$ which is set-theoretically the subset $\bigcup_{J} \Omega_{J}\left(G_{\bullet}\right) \times \Omega_{J^{\prime}}\left(G_{\bullet}^{\prime}\right)$ of $\Delta(r-1, K)$ (here $G_{\bullet}$ and $G_{\bullet}^{\prime}$ are the induced flags on $K$, and $J$ runs through all subsets of $[n-1]$ of rank $r-1)$. The natural map

$$
\mathcal{T}\left(\bigcup_{J} \Omega_{J}\left(G_{\bullet}\right) \times \Omega_{J^{\prime}}\left(G_{\bullet}^{\prime}\right)\right) \rightarrow \operatorname{Gr}(r, W) \times \operatorname{Gr}(r, W)
$$

has image $\bigcup_{I: 1 \in I} \Omega_{I}\left(F_{\bullet}\right) \times \Omega_{I^{\prime}}\left(F_{\bullet}^{\prime}\right)$ and is birational over $\bigcup_{I: 1 \in I} \Omega_{I}^{o}\left(F_{\bullet}\right) \times \Omega_{I^{\prime}}^{o}\left(F_{\bullet}^{\prime}\right) \subseteq$ $\operatorname{Gr}(r, W) \times \operatorname{Gr}(r, W)$ (the association between $I$ and $J$ is $I=\{1\} \cup\{a+1: a \in J\}$ ). The proof is now complete.

\subsection{Tangent spaces. Let}

$$
\Delta_{+}^{o}=\Delta_{+}-\Delta_{-} .
$$

It is easy to see that $\Delta_{+}^{o}$ is a smooth open dense subset of $\Delta_{+}$.

Let $(A, B) \in \Delta_{+}$. Recall that $\beta: W \rightarrow W$ is the projection to $K$ corresponding to the decomposition $W=L \oplus K$. Then $\beta(A) \subset B$ and $\beta$ induces a morphism $W / A \rightarrow W / B$.

Lemma 5.3. For $(A, B) \in \Delta_{+}^{o}$, the induced map $\beta: W / A \rightarrow W / B$ is an isomorphism. The induced map $\beta: A \rightarrow B$ is singular with kernel of rank 1 .

Proof. Consider the natural map $p: K \rightarrow W \rightarrow W / B$ (inclusion followed by projection). The rank of the kernel is $\operatorname{rk}(K \cap B)=\operatorname{rk}(B)-1$ (since $(A, B) \notin \Delta_{-}$, $B \nsubseteq K)$, and hence the rank of the image is $\operatorname{rk}(K)+1-\operatorname{rk}(B)=\operatorname{rk}(W / B)$. Therefore the composite $K \rightarrow W / B$ is surjective. But $\beta$ induces a surjective map $W \rightarrow K$. The composite $W \stackrel{\beta}{\rightarrow} K \stackrel{p}{\rightarrow} W / B$ is surjective and $A$ is in its kernel. So $\beta$ induces a surjective (hence isomorphism) map $W / A \rightarrow W / B$.

Since $\operatorname{ker}(\beta)=L$ and $L \subset A$, the second assertion is clear.

Lemma 5.4. The tangent space to the scheme $\Delta_{\beta}$ at $(A, B)$ is the vector subspace of the tangent space of $\operatorname{Gr}(r, W) \times \operatorname{Gr}(r, W)$ at $(A, B)$ given by pairs of maps $A \stackrel{\Gamma_{A}}{\rightarrow}$ $W / A, B \stackrel{\Gamma_{B}}{\longrightarrow} W / B$ such that the following diagram commutes:

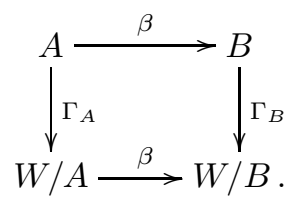

Proof. Choose splittings $W=A \oplus W / A$ and $W=B \oplus W / B$. For $\psi \in W / A$, write $\beta(0 \oplus \psi)=b(\psi) \oplus \beta(\psi)$ (the last term arises from the natural map $W / A \rightarrow W / B$ ).

Suppose $\Gamma_{A}: A \rightarrow W / A$ and $\Gamma_{B}: B \rightarrow W / B$ are deformations of $A$ and $B$, respectively. This means that the deformed $A$ (similarly $B$ ) is the $\mathbb{C}[\epsilon] /\left(\epsilon^{2}\right)$ span of elements of the form $a \oplus \epsilon \Gamma_{A}(a)$. The condition that this deformation stays inside $\Delta_{\beta}$ is that $\beta\left(a \oplus \epsilon \Gamma_{A}(a)\right)$ should be in the $\mathbb{C}[\epsilon] /\left(\epsilon^{2}\right)$ span of terms of the form $b \oplus \epsilon \Gamma_{B}(b)$. A typical element in this span looks like $\left(b+\epsilon b^{\prime}\right) \oplus \epsilon \Gamma_{B}(b)$ with $b, b^{\prime} \in B$. Write $\beta\left(a \oplus \epsilon \Gamma_{A}(a)\right)=\left(b+\epsilon b^{\prime}\right) \oplus \epsilon \Gamma_{B}(b)$. This forces $b=\beta(a)$, and hence reading the $\epsilon$ term and its component in $W / B$, we get the desired commutativity. 


\section{6. $S$-BUndLES AND THE DIAGONAL}

Let $T$ be an ES-vector bundle of rank $r+k$ and degree $k(g-1)$ on $\mathbb{P}^{1}$. Fix isomorphisms $G_{j}: T_{p_{j}} \rightarrow W$ and $H_{j}: T_{q_{j}} \rightarrow W$.

We therefore have maps

$$
\operatorname{Gr}(0, r, T) \rightarrow \prod_{j=1}^{g}\left(\operatorname{Gr}\left(r, T_{p_{j}}\right) \times \operatorname{Gr}\left(r, T_{q_{j}}\right)\right) \rightarrow \prod_{j=1}^{g}(\operatorname{Gr}(r, W) \times \operatorname{Gr}(r, W)) .
$$

Call the composite $\Phi=\Phi(G, H)$. Fix $W=L \oplus K$ and the projection $\beta: W \rightarrow W$ as in Section 5. Define $\gamma_{j}: T_{p_{j}} \rightarrow T_{q_{j}}$ using the diagram

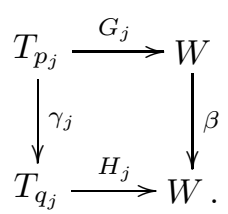

Proposition 6.1. (a) For generic $(G, H)$,

$$
\Phi(G, H)^{-1}\left(\prod_{j=1}^{g} \Delta_{+}\right)=\Phi(G, H)^{-1}\left(\prod_{j=1}^{g} \Delta_{+}^{o}\right) .
$$

(b) $\Phi(G, H)^{-1}\left(\prod_{j=1}^{g} \Delta_{+}\right)$is in bijection with the set of singular subbundles of degree 0 and rank $r$ of $(T, \gamma)$ considered in Section 4 .

(c) For generic $(G, H), \Phi(G, H)^{-1}\left(\prod_{j=1}^{g} \Delta_{+}^{o}\right)$ is reduced and of the expected dimension 0 .

Proof. Item (b) follows easily from the definitions. For (c), the expected dimension is

$$
\operatorname{dim} \operatorname{Gr}(0, r, T)-g \operatorname{dim} \operatorname{Gr}(r, W)=r k+k(g-1) r-g r k=0 .
$$

Note that $\operatorname{GL}(W) \times \operatorname{GL}(W)$ acts transitively on $\operatorname{Gr}(r, W) \times \operatorname{Gr}(W)$. Therefore the group $\prod_{j=1}^{g}(\mathrm{GL}(W) \times \mathrm{GL}(W))$ acts transitively on $\prod_{j=1}^{g}(\operatorname{Gr}(r, W) \times \operatorname{Gr}(r, W))$. We now recall that $\Delta_{+}^{o}$ is smooth and connected. Hence, by Kleiman's transversality theorem (cf. [Kl], $[\mathrm{Fu}] \S \mathrm{B} .9 .2$ ), for generic $\tau \in \prod_{j=1}^{g}(\mathrm{GL}(W) \times \mathrm{GL}(W))$, $\Phi^{-1} \tau^{-1}\left(\prod_{j=1}^{g} \Delta_{+}^{o}\right)$ is reduced and of the expected dimension $=0$. Notice that the action of $\tau$ just modifies the maps $G$ and $H$. Therefore the assertion (c) follows. The equality in (a) holds because if $S$ is a non-empty subset of $\{1, \ldots, g\}$, the expected dimension of (any component of) $\Phi(G, H)^{-1}\left(\prod_{j=1}^{g} A_{j}\right)$ is negative where $A_{j}=\Delta_{+}$if $j \notin S$ and $A_{j}=\Delta_{+} \cap \Delta_{-}$if $j \in S$.

Now let $G$ and $H$ be generic and let

$$
m=\left|\Phi^{-1}\left(\prod_{j=1}^{g} \Delta_{+}^{o}\right)\right|,\left\{V^{(1)}, \ldots, V^{(m)}\right\}=\Phi^{-1}\left(\prod_{j=1}^{g} \Delta_{+}^{o}\right) .
$$

Let $Q^{(a)}=T / V^{(a)}$ for $a=1, \ldots, m$. We will now endow $V^{(a)}$ and $Q^{(a)}$ with $S$ bundle structure using the diagrams, obtaining $S$-bundles $\left(V^{(a)}, \tau^{(a)}\right)$ and 
$\left(Q^{(a)}, \theta^{(a)}\right)$, respectively:

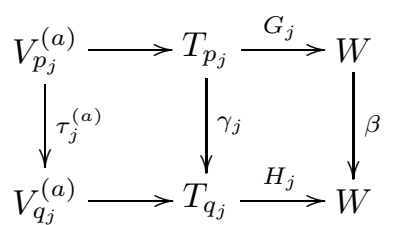

and

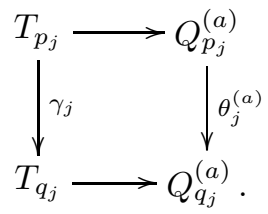

Proposition 6.2. The inequality $m \geq M(r, k, g)$ holds.

The proof of Proposition 6.2 will appear in Section 8 . It will eventually be shown that $m=M(r, k, g)$.

Lemma 6.3. The tangent space at $V^{(a)}$ to $\Phi^{-1}\left(\prod_{j=1}^{g} \Delta_{+}^{o}\right)$ is

$$
\underline{\operatorname{Hom}}\left(\left(V^{(a)}, \tau^{(a)}\right),\left(Q^{(a)}, \theta^{(a)}\right)\right) \text {. }
$$

Proof. The tangent space to $\operatorname{Gr}(0, r, T)$ at $V^{(a)} \subseteq T$ is $\operatorname{Hom}\left(V^{(a)}, T / V^{(a)}\right)$ by Grothendieck's theory of quot schemes.

For $j=1, \ldots, g$, let $\Phi_{j}$ be the composition (where the last map is the projection to the $j$ th factor):

$$
\begin{aligned}
\operatorname{Gr}(0, r, T) & \rightarrow \prod_{j=1}^{g} \operatorname{Gr}\left(r, T_{p_{j}}\right) \times \operatorname{Gr}\left(r, T_{q_{j}}\right) \\
& \prod\left(\stackrel{\left.G_{j}, H_{j}\right)}{\longrightarrow} \prod_{j=1}^{g} \operatorname{Gr}(r, W) \times \operatorname{Gr}(r, W) \rightarrow \operatorname{Gr}(r, W) \times \operatorname{Gr}(r, W) .\right.
\end{aligned}
$$

An element $\Gamma \in \operatorname{Hom}\left(V^{(a)}, T / V^{(a)}\right)$ is in the tangent space of $\Phi^{-1}\left(\prod_{j=1}^{g} \Delta_{+}\right)$at $V^{(a)}$ if and only for each $j=1, \ldots, g,\left(\Phi_{j}\right)_{*}(\Gamma)$ is in the tangent space of $\Delta_{+}^{o}$ at $\left(G_{j}\left(V_{p_{j}}^{(a)}\right), H_{j}\left(V_{q_{j}}^{(a)}\right)\right)$. By Lemma 5.4, this condition implies that, as desired, $\Gamma \in \underline{\operatorname{Hom}}\left(\left(V^{(a)}, \tau^{(a)}\right),\left(Q^{(a)}, \theta^{(a)}\right)\right)$.

Lemma 6.4. (i) $\underline{\operatorname{Hom}}\left(\left(V^{(a)}, \tau^{(a)}\right),\left(Q^{(b)}, \theta^{(b)}\right)\right) \neq 0$ if and only if $a \neq b$.

(ii) The coherent sheaves underlying the $S$-bundles $\left(Q^{(a)}, \theta^{(a)}\right), a=1, \ldots, m$, are vector bundles on $C$ of degree $k(g-1)$.

(iii) For $a=1, \ldots, m$ and $j=1, \ldots, g$, the linear map of vector spaces $\tau_{j}^{(a)}$ : $V_{p_{j}}^{(a)} \rightarrow V_{q_{j}}^{(a)}$ is singular with a rank 1 kernel.

(iv) The vector bundles $V^{(a)}$ (resp. $\left.Q^{(a)}\right)$ for $a=1, \ldots, m$ on $\mathbb{P}^{1}$ are ES and are hence isomorphic. In particular, $V^{(a)} \stackrel{\sim}{\rightarrow} \mathcal{O}_{\mathbb{P}^{1}}^{\oplus r}$.

Proof. This proof is modeled after a similar proof in [Be1]. By Lemma 6.3, the tangent space at $V^{(a)}$ to $\Phi^{-1}\left(\Delta_{+}\right)$is $\underline{\operatorname{Hom}}\left(\left(V^{(a)}, \tau^{(a)}\right),\left(Q^{(a)}, \theta^{(a)}\right)\right)$ which is consequently 0 , because of Proposition 6.1. 
If $a \neq b$, consider the non-zero (!) composite $V^{(a)} \rightarrow T \rightarrow Q^{(b)}$ (inclusion followed by projection). This composite belongs to $\underline{\operatorname{Hom}}\left(\left(V^{(a)}, \tau^{(a)}\right),\left(Q^{(b)}, \theta^{(b)}\right)\right)$ (the property of being a morphism of $S$-bundles follows from diagrams (6.2) and (6.3)). This proves (i).

Now $V^{(a)} \in \Phi^{-1}\left(\Delta_{+}^{o}\right)$, and therefore $\theta_{j}^{(a)}: Q_{p_{j}}^{(a)} \rightarrow Q_{q_{j}}^{(a)}$ is an isomorphism (see Lemma 5.3). So (ii) follows immediately.

Assertion (iii) follows because $L \subseteq \operatorname{ker}(\beta)$. Assertion (iv) follows from Proposition 4.1 and Kleiman's transversality theorem.

\section{7. $S$-Bundes And the Determinant of COHOMOlogy}

Let $V=\mathcal{O}_{\mathbb{P}^{1}}^{r}$ and let $Q$ be an ES-bundle of degree $k(g-1)$ and rank $k$. Let $(V, \tau)$ and $(Q, \theta)$ be $S$-bundles on $C$ so that $\theta_{j}$ is an isomorphism for $j=1, \ldots, g$. It follows that the coherent sheaf underlying $(Q, \theta)$ is an honest vector bundle $\tilde{Q}$ on $C$ of rank $k$ and degree $k(g-1)$.

Define $\mathcal{A}=\mathcal{A}(\tau, \theta)$, a coherent sheaf on $C$, by the following exact sequence of sheaves:

$$
0 \rightarrow \mathcal{A} \rightarrow f_{*} \mathcal{H} \operatorname{Hom}(V, Q) \stackrel{\Sigma}{\rightarrow} \bigoplus_{j=1}^{g} \operatorname{Hom}\left(V_{p_{j}}, Q_{q_{j}}\right) \mid r_{j} \rightarrow 0
$$

where the last sheaf is a skyscraper sheaf at $r_{j}$ and

$$
\Sigma(\Gamma)=\theta_{j} \circ \Gamma_{p_{j}}-\Gamma_{q_{j}} \circ \tau_{j} \in \operatorname{Hom}\left(V_{p_{j}}, Q_{p_{j}}\right) .
$$

Since $\theta_{j}$ are assumed to be isomorphisms, the map $\Sigma$ is surjective. If $\tau$ and $\theta$ vary in families so that $\theta_{j}$ are isomorphisms (on each fiber over the parameter space), then $\mathcal{A}(\tau, \theta)$ is flat over the parameter space. This is because the kernel of a surjective map of flat modules is flat (long exact sequence in Tor!).

Since $f$ is a finite morphism,

$$
\begin{aligned}
\chi(C, \mathcal{A}) & =\chi\left(C, f_{*} \mathcal{H o m}(V, Q)\right)-g r k \\
& =\chi\left(\mathbb{P}^{1}, \mathcal{H} \operatorname{om}(V, Q)\right)-g r k=k r(g-1)+k r-g r k=0 .
\end{aligned}
$$

So there is then a canonical section (note that for a coherent sheaf $A$ on $\mathbb{P}^{1}, \mathcal{D}(A)=$ $\left.\mathcal{D}\left(f_{*} A\right)\right)$

$$
\sigma(\tau, \theta) \in \mathcal{D}(\mathcal{A}(\tau, \theta)) \stackrel{\sim}{\rightarrow} \mathcal{D}(\mathcal{H o m}(V, Q)) \otimes \bigotimes_{j=1}^{g} \operatorname{det}\left(\operatorname{Hom}\left(V_{p_{j}}, Q_{q_{j}}\right)\right)^{*}
$$

We note the following properties of this construction.

Lemma 7.1. (1) $H^{0}(C, \mathcal{A})=\underline{\operatorname{Hom}}((V, \tau),(Q, \theta))$.

(2) $\sigma(\tau, \theta) \neq 0$ if and only if $\underline{\operatorname{Hom}}((V, \tau),(Q, \theta))=0$.

(3) If the $\tau_{j}$ are isomorphisms for $j=1, \ldots, g$ and $\tilde{V}$ the locally free coherent sheaf underlying $(V, \tau)$, then we have a natural isomorphism of sheaves on $C: \mathcal{A} \rightarrow \mathcal{H o m}(\tilde{V}, \tilde{Q})$.

7.1. Geometric $S$-bundles. Now consider the $S$-bundles obtained from geometry in Section 6 (Lemma 6.4). By Lemma 6.4(iv), the vector bundles $V^{(a)}$ on $\mathbb{P}^{1}$ are all isomorphic to the vector bundle $V=\mathcal{O}_{\mathbb{P}^{1}}^{\oplus r}$, and the bundles $Q^{(a)}$ are all isomorphic to $Q$ where $Q$ is the unique ES-bundle of the degree and rank of $Q^{(a)}$. We choose isomorphisms $\left(V^{(a)}, \tau^{(a)}\right) \stackrel{\sim}{\rightarrow}\left(V, \tilde{\tau}^{(a)}\right)$ and $\left(Q^{(a)}, \theta^{(a)}\right) \stackrel{\sim}{\rightarrow}\left(Q, \tilde{\theta}^{(a)}\right)$ for $a=1, \ldots, m$, for suitable $\tilde{\tau}$ and $\tilde{\theta}$. 
From Lemma 6.4 we know that the $\tilde{\theta}_{j}^{(b)}$ are isomorphisms for $j=1, \ldots, g$. Consider,

$$
\sigma\left(\tilde{\tau}^{(a)}, \tilde{\theta}^{(b)}\right) \in \mathcal{D}\left(\mathcal{A}\left(\tilde{\tau}^{(a)}, \tilde{\theta}^{(b)}\right)\right) \stackrel{\sim}{\rightarrow} \mathcal{D}(\mathcal{H o m}(V, Q)) \otimes \bigotimes_{j=1}^{g} \operatorname{det}\left(\operatorname{Hom}\left(V_{p_{j}}, Q_{q_{j}}\right)\right)^{*}
$$

We may think of this as an $m \times m$ matrix with entries in the one dimensional vector space $\mathcal{D}(\mathcal{H o m}(V, Q)) \otimes \bigotimes_{j=1}^{g} \operatorname{det}\left(\operatorname{Hom}\left(V_{p_{j}}, Q_{q_{j}}\right)\right)^{*}$. By Lemma 6.4 and Lemma $7.1(2)$, the $(a, b)$ entry is non-zero exactly when $a=b$. Hence the matrix is non-singular.

Find maps $\lambda_{j}^{(a)}(t) \in \operatorname{Hom}\left(V_{p_{j}}, V_{q_{j}}\right)=\operatorname{Hom}\left(\mathbb{C}^{r}, \mathbb{C}^{r}\right)$ (using the identification $V \rightarrow \mathcal{O}_{\mathbb{P}^{1}}^{\oplus r}$ ) so that for $j=1, \ldots, g$,

(1) $\lambda_{j}^{(a)}(0)=\tilde{\tau}_{j}^{(a)}$

(2) $\operatorname{det}\left(\lambda_{j}^{(a)}(t)\right)=t$.

(we may find such matrices using Jordan canonical forms because the kernel of $\tilde{\tau}_{j}^{(a)}$ is one dimensional for $a=1, \ldots, m$ and $\left.j=1, \ldots, g\right)$. Therefore, for $a, a^{\prime} \in$ $\{1, \ldots, m\}, \operatorname{det}\left(\lambda_{j}^{(a)}(t)\right)=\operatorname{det}\left(\lambda_{j}^{\left(a^{\prime}\right)}(t)\right)$ and these numbers are non-zero if $t \neq 0$. Consider the $m \times m$ matrix formed by

$$
\sigma\left(\lambda^{(a)}(t), \tilde{\theta}^{(b)}\right) \in \mathcal{D}\left(\mathcal{A}\left(\lambda^{(a)}(t), \tilde{\theta}^{(b)}\right)\right) \stackrel{\sim}{\rightarrow} \mathcal{D}(\mathcal{H o m}(V, Q)) \otimes \bigotimes_{j=1}^{g} \operatorname{det}\left(\operatorname{Hom}\left(V_{p_{j}}, Q_{q_{j}}\right)\right)^{*}
$$

This matrix is going to be non-singular for values of $t$ in a sufficiently small Zariski neighborhood of $t=0$. Let $\epsilon \neq 0$ be one such value for $t$. Let $\tilde{V}^{(a)}$ be the locally free coherent sheaf underlying $\left(V, \lambda^{(a)}(\epsilon)\right)$. Let $\tilde{Q}^{(b)}$ be the coherent sheaf on $C$ underlying $\left(Q, \tilde{\theta}^{(b)}\right)$. From Lemma 6.4 , we see that $\tilde{Q}^{(b)}$ is locally free of degree $k(g-1)$. According to Lemma 3.2, $\tilde{V}^{(a)}$ have isomorphic determinant line bundles of degree 0 for $a=1, \ldots, m$.

Lemma 7.2. The tuple $\left(\left(\tilde{V}^{(1)}\right)^{*}, \ldots,\left(\tilde{V}^{(m)}\right)^{*} ; \tilde{Q}^{(1)}, \ldots, \tilde{Q}^{(m)}\right)$ is an $(m, r, k)$-frame on the rational nodal curve $C$.

Together with Lemma 2.5 and Proposition 2.3. this would conclude the proof of the strange duality conjecture for generic curves if we can show that the identifications that we made here are compatible with the determinant operation that went with the definition of the frame.

7.2. The basic compatibility verification. Let $(V, \tau)$ and $(V, \eta)$ be $S$-bundles such that $V$ is of degree 0 and rank $r$. Let $\tilde{V}^{1}$ and $\tilde{V}_{2}$ be the underlying coherent sheaves on $C$. Assume that $\tau_{j}$ and $\eta_{j}$ are isomorphisms for $j=1, \ldots, g$ (so $\tilde{V}^{1}$ and $\tilde{V}^{2}$ are vector bundles on $\left.C\right)$. Assume further that $\operatorname{det}\left(\tilde{V}^{1}\right) \stackrel{\sim}{\rightarrow} \operatorname{det}\left(\tilde{V}^{2}\right)$. Let $(Q, \theta)$ and $(Q, \delta)$ be $S$-bundles such that $\theta_{j}$ and $\delta_{j}$ are isomorphisms, $j=1, \ldots, g$, and $\tilde{Q}_{1}$ and $\tilde{Q}_{2}$ are the corresponding vector bundles on $C$. Assume that $Q$ is of rank 
$k$ and degree $k(g-1)$. We need to verify that the following diagram commutes: (7.2)

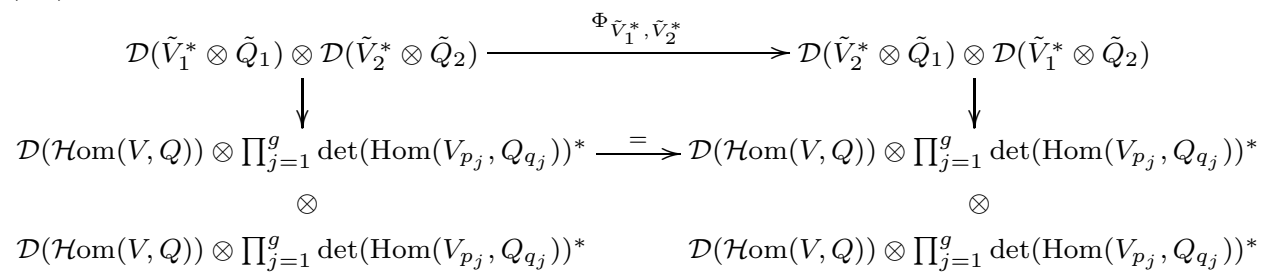

For this we first consider two natural exact sequences associated to $\tilde{Q}_{1}$ and $\tilde{Q}_{2}$. The one associated to $\tilde{Q}_{1}$ is

$$
0 \rightarrow \tilde{Q}_{1} \rightarrow f_{*} Q \stackrel{\alpha}{\rightarrow} \bigoplus_{j=1}^{g} Q_{q_{j}} \mid r_{j} \rightarrow 0
$$

where $\alpha_{j}: Q_{p_{j}} \oplus Q_{q_{j}} \rightarrow Q_{q_{j}}$ takes $\left(q_{1}, q_{2}\right) \rightarrow q_{2}-\theta_{j}\left(q_{1}\right)$. Notice that for $a=1,2$ there are isomorphisms

$$
f_{*} Q \otimes \tilde{V}_{a}^{*} \rightarrow f_{*}\left(V^{*} \otimes Q\right)=f_{*} \mathcal{H o m}(V, Q),
$$

and

$$
\left(Q_{q_{j}} \mid r_{j}\right) \otimes \tilde{V}_{a}^{*}=Q_{q_{j}} \otimes V_{p_{j}}^{*}\left|r_{j}=\operatorname{Hom}\left(V_{p_{j}}, Q_{q_{j}}\right)\right| r_{j} .
$$

From Section 2.1, recall the second observation and the compatibility under exact sequences in $(I, J)$. We now see that diagram (7.2) commutes.

\section{Proof of Proposition 6.2}

8.1. Conformal blocks. We recall the following.

(1) Irreducible polynomial representations of $U(r)$ are parameterized by weakly decreasing sequences of non-negative integers $\lambda=\left(\lambda_{1} \geq \lambda_{2} \geq \cdots \geq \lambda_{r}\right) \in$ $\mathbb{Z}^{r}$. These restrict to irreducible representations $\bar{\lambda}$ of $S U(r)$. For such a $\lambda$, set $|\lambda|=\sum_{a=1}^{r} \lambda_{a} \in \mathbb{Z}$.

(2) $\left(\lambda_{1}, \lambda_{2}, \ldots, \lambda_{r}\right)$ and $\left(\mu_{1}, \mu_{2}, \ldots, \mu_{r}\right)$ restrict to give the same irreducible representation of $S U(r)$ if $\lambda_{a}-\mu_{a}$ is a constant for $a=1, \ldots, r$. The congruence class of $|\bar{\mu}|=\sum_{a} \mu_{a}(\bmod r)$ is therefore a well defined $\mathbb{Z} / r \mathbb{Z}$ "invariant" of the representation $\bar{\mu}$ of $S U(r)$.

(3) The dual of a representation $\bar{\mu}$ of $S U(r)$ is denoted by $\bar{\mu}^{*}$ and equals $\left(\mu_{1}-\right.$ $\left.\mu_{r}, \mu_{1}-\mu_{r-1}, \ldots, \mu_{1}-\mu_{2}, 0\right)$.

(4) A representation $\bar{\lambda}$ of $S U(r)$ with $\lambda=\left(\lambda_{1}, \lambda_{2}, \ldots, \lambda_{r}\right)$ is said to have level $\leq k$ if $\lambda_{1}-\lambda_{r} \leq k$.

Given irreducible representations $\bar{\mu}^{1}, \ldots, \bar{\mu}^{s}$ of level $\leq k$ of $S U(r)$, we obtain the dimensions of spaces of conformal blocks $N_{g}^{(k)}\left(\bar{\mu}^{1}, \ldots, \bar{\mu}^{s}\right)$ (as in [B3]). It is known that $N_{g}^{(k)}(\emptyset)=M(r, k, g)$ (cf. [BL, [Fa2] and [KNR]). There is a similar identification [Pa] of $N_{g}^{(k)}\left(\bar{\mu}^{1}, \ldots, \bar{\mu}^{s}\right)$ with the rank of the space of sections of the $k$-fold tensor product of a certain line bundle on a moduli space of parabolic bundles over a smooth projective curve of genus $g$ (with $s$ marked points and associated parabolic data $\left.\bar{\mu}^{1}, \ldots, \bar{\mu}^{s}\right)$. 
The factorization theorem of Tsuchiya, Ueno, and Yamada (see [TUY] and [B3]) gives

$$
N_{g}^{(k)}\left(\bar{\mu}^{1}, \ldots, \bar{\mu}^{s}\right)=\sum_{\bar{\nu}} N_{g-1}^{(k)}\left(\bar{\mu}^{1}, \ldots, \bar{\mu}^{s}, \bar{\nu}, \bar{\nu}^{*}\right)
$$

(where $\bar{\nu}$ runs through all irreducible representations of $S U(r)$ of level $\leq k$ ). This formula corresponds to a degeneration of smooth genus $g$ curves with $s$ marked points into a nodal curve of arithmetic genus $g$ and its subsequent normalization to a genus $g-1$ curve (this adds two new marked points).

Repeated application of this formula gives

$$
N_{g}^{(k)}(\emptyset)=\sum_{\bar{\mu}^{1}, \ldots, \bar{\mu}^{g}} N_{0}^{(k)}\left(\bar{\mu}^{1},\left(\bar{\mu}^{1}\right)^{*}, \bar{\mu}^{2},\left(\bar{\mu}^{2}\right)^{*}, \ldots, \bar{\mu}^{g},\left(\bar{\mu}^{g}\right)^{*}\right)
$$

where $\bar{\mu}^{1}, \ldots, \bar{\mu}^{g}$ vary over all irreducible representations of $S U(r)$ of level $\leq k$.

The Verlinde algebra for $S U(r)$ at level $\leq k$ is given by the rule

$$
\bar{\mu}^{1} \cdot \bar{\mu}^{2} \cdots \bar{\mu}^{s}=\sum_{\bar{\nu}} N_{0}^{(k)}\left(\bar{\mu}^{1}, \ldots, \bar{\mu}^{s}, \bar{\nu}\right) \bar{\nu}^{*}
$$

where $\bar{\nu}$ runs through all irreducible representations of $S U(r)$ of level $\leq k$.

8.2. Generalized Gromov-Witten numbers. Fix positive integers $r$ and $k$ and set $n=r+k$. Suppose $I^{1}, \ldots, I^{s}$ are subsets of $[n]=\{1, \ldots, n\}$ each of cardinality $r$.

Let $\rho_{1}, \ldots, \rho_{s}$ be a set of distinct points on $\mathbb{P}^{1}$. Let $T$ be an evenly split (ES) bundle of degree $-D$ and rank $n$ (recall the definition of ES-bundles given in Section 4 ). Choose generic complete flags $E_{\bullet}^{\rho_{j}}$ on the fibers $T_{\rho_{j}}$ for $j=1, \ldots, s$.

Define $\left\langle\omega_{I^{1}}, \omega_{I^{2}}, \ldots, \omega_{I^{s}}\right\rangle_{d, D}$ to be the number of subbundles (0 if this number is infinite) $V$ of $T$ of degree $-d$ and rank $r$, such that for $j=1, \ldots, s$, the point $V_{\rho_{j}} \in \operatorname{Gr}\left(r, T_{\rho_{j}}\right)$ lies in the Schubert cell $\Omega_{I^{j}}^{o}\left(E_{\bullet^{\rho_{j}}}\right)$. By Kleiman's transversality theorem the space of such $V$ is equidimensional of dimension (using Proposition 4.1)

$$
\operatorname{dim}(\operatorname{Gr}(d, r, T))-\sum_{j=1}^{s} \operatorname{codim}\left(\omega_{I^{j}}\right)=r(n-r)+d n-D r-\sum_{j=1}^{s} \operatorname{codim}\left(\omega_{I^{j}}\right) .
$$

If $D=0$, it is easy to see that the above definition gives the structure coefficients in the small quantum cohomology of $\operatorname{Gr}(r, n)$ (for example see [FP, Section 10). Here we use the standard bijection between subbundles of $\mathcal{O}_{\mathbb{P}^{1}}^{\oplus n}$ of rank $r$ and degree $-d$ and maps $\mathbb{P}^{1} \rightarrow \operatorname{Gr}(r, n)$ of degree $d$. The numbers when $D \neq 0$ can be recovered from the small quantum cohomology structure constants by using shift operations (see Proposition A.3).

One also notes that if $L=\{n-r+1, n-r+2, \ldots, n\}$, then

$$
\left\langle\omega_{I^{1}}, \omega_{I^{2}}, \ldots, \omega_{I^{s}}\right\rangle_{d, D}=\left\langle\omega_{I^{1}}, \omega_{I^{2}}, \ldots, \omega_{I^{s}}, \omega_{L}\right\rangle_{d, D} .
$$

This is because we are imposing an open dense condition at the $(s+1)$ th point.

8.3. Proof of Proposition 6.2. We return to the notation and setting of Proposition 6.2. We introduce a new piece of notation relating Schubert cells in $\operatorname{Gr}(r, n)$ and representations of $U(r)$ (equivalently, GL $(r)$ ). To an $r$-element subset $I=$ $\left\{i_{1}<\cdots<i_{r}\right\}$ of $[n]=\{1, \ldots, n\}$ we associate a weakly decreasing sequence of non-negative integers

$$
I \mapsto \lambda(I)=\left(\lambda_{1} \geq \lambda_{2} \geq \cdots \geq \lambda_{r}\right) \in \mathbb{Z}_{\geq 0}^{r},
$$


where $\lambda_{a}=n-r+a-i_{a}, a=1, \ldots, r$. We note the obvious:

Lemma 8.1. (1) As I runs through all r-element subsets of $[n]$ with $1 \in I$, $\bar{\lambda}(I)$ runs (exactly once) through all irreducible representations of $S U(r)$ of level $\leq k=n-r$

(2) For I as in (1), $\bar{\lambda}\left(I^{\prime}\right)=\bar{\lambda}(I)^{*}$.

We return to the proof of Proposition 6.2. Recall that by Proposition 5.1, $\Delta_{+}$ degenerates into a subscheme with support

$$
\bigcup_{I: 1 \in I} \Omega_{I}\left(F_{\bullet}\right) \times \Omega_{I^{\prime}}\left(F_{\bullet}^{\prime}\right) \subseteq \operatorname{Gr}(r, W) \times \operatorname{Gr}(r, W) .
$$

By Proposition [5.1, the degeneration agrees as a scheme with the above (reduced) subscheme over the smooth open dense $\bigcup_{I: 1 \in I} \Omega_{I}^{o}\left(F_{\bullet}\right) \times \Omega_{I^{\prime}}^{o}\left(F_{\bullet}^{\prime}\right)$.

We may assume by modifying the maps $G_{j}, H_{j}$ in diagram (6.1) that any $\Phi^{-1}\left(\prod_{j=1}^{g} \Omega_{I^{j}}\left(F_{\bullet}\right) \times \Omega_{\left(I^{j}\right)^{\prime}}\left(F_{\bullet}^{\prime}\right)\right)$ is smooth of the expected dimension zero (using Kleiman's transversality theorem). We may assume that any point in it is actually in $\Phi^{-1}\left(\prod_{j=1}^{g} \Omega_{I^{j}}^{o}\left(F_{\bullet}\right) \times \Omega_{\left(I^{j}\right)^{\prime}}^{o}\left(F_{\bullet}^{\prime}\right)\right.$ ) (in fact if $U \subseteq \prod_{j=1}^{g} \Omega_{I^{j}}^{o}\left(F_{\bullet}\right) \times \Omega_{\left(I^{j}\right)^{\prime}}^{o}\left(F_{\bullet}^{\prime}\right)$ is any non-empty open subset, then for generic $(G, H), \Phi^{-1}(U)$ equals $\Phi^{-1}\left(\prod_{j=1}^{g} \Omega_{I^{j}}\left(F_{\bullet}\right)\right.$ $\left.\times \Omega_{\left(I^{j}\right)^{\prime}}\left(F_{\bullet}^{\prime}\right)\right)$.)

The number of points in $\Phi^{-1}\left(\prod_{j=1}^{g} \Omega_{I^{j}}^{o}\left(F_{\bullet}\right) \times \Omega_{\left(I^{j}\right)^{\prime}}^{o}\left(F_{\bullet}^{\prime}\right)\right)$ is clearly

$$
\left\langle\omega_{I^{1}}, \ldots, \omega_{I^{g}}, \omega_{\left(I^{1}\right)^{\prime}}, \ldots, \omega_{\left(I^{g}\right)^{\prime}}\right\rangle_{0,-k(g-1)} .
$$

This implies that for some $\left(t_{2}, \ldots, t_{n}\right)$ with $t_{i} \neq 0$,

$$
\Phi^{-1}\left(1 \times \phi_{n}\left(t_{n}\right)\right)\left(1 \times \phi_{n-1}\left(t_{n-1}\right)\right) \ldots\left(1 \times \phi_{2}\left(t_{2}\right)\right) \Delta_{+}
$$

has at least

$$
m_{0}=\sum^{\prime}\left\langle\omega_{I^{1}}, \ldots, \omega_{I^{g}}, \omega_{\left(I^{1}\right)^{\prime}}, \ldots, \omega_{\left(I^{g}\right)^{\prime}}\right\rangle_{0,-k(g-1)}
$$

smooth and reduced points, where the sum is over all sequences of subsets $\left(I^{1}, \ldots\right.$, $\left.I^{g}\right)$ of $[n]$ with $r$ elements, each of which contains 1.

Hence for generic $G$ and $H$ (by absorbing 1 and $\phi_{n}\left(t_{n}\right) \circ \ldots \phi\left(t_{2}\right)$ in the maps $G$ and $H)$, we see that $\Phi(G, H)^{-1}\left(\prod_{j=1}^{g} \Delta_{+}^{o}\right)$ has at least $m_{0}$ isolated and reduced points (the passage from $\Delta_{+}$to $\Delta_{+}^{o}$ is clear because the intersection is over smooth points of $\left.\Delta_{+}\right)$. So $m \geq m_{0}$.

The following proposition follows from a theorem of Witten and will be proved in Section 8.5.1.

Proposition 8.2. Suppose $I^{1}, \ldots, I^{g}$ are $r$-element subsets of $[n]$ and $\mu^{j}=\lambda\left(I^{j}\right)$ for $j=1, \ldots, g$. Then

$$
\left\langle\omega_{I^{1}}, \ldots, \omega_{I^{s}}, \omega_{\left(I^{1}\right)^{\prime}}, \ldots, \omega_{\left(I^{g}\right)^{\prime}}\right\rangle_{0,-k(g-1)}=N_{0}^{(k)}\left(\bar{\mu}^{1},\left(\bar{\mu}^{1}\right)^{*}, \bar{\mu}^{2},\left(\bar{\mu}^{2}\right)^{*}, \ldots, \bar{\mu}^{g},\left(\bar{\mu}^{g}\right)^{*}\right) .
$$

From Proposition 8.2 and Lemma 8.1(1), it follows that

$$
m_{0}=\sum_{\bar{\mu}^{1}, \ldots, \bar{\mu}^{g}} N_{0}^{(k)}\left(\bar{\mu}^{1},\left(\bar{\mu}^{1}\right)^{*}, \bar{\mu}^{2},\left(\bar{\mu}^{2}\right)^{*}, \ldots, \bar{\mu}^{g},\left(\bar{\mu}^{g}\right)^{*}\right)
$$

where $\bar{\mu}^{1}, \ldots, \bar{\mu}^{g}$ vary over all irreducible representations of $S U(r)$ of level $\leq k$. By equation (8.1), one sees now that $m_{0}=M(r, k, g)$ and this concludes the proof of Proposition 6.2. 
8.4. Examples. We first consider the case $k=1$. In this case in the formula $(\ddagger)$ $n=r+1$ and $I^{1}, \ldots, I^{g}$ are subsets of $[r+1]=\{1, \ldots, r+1\}$ each with $r$ elements and containing 1 . There are $r$ choices for each $I^{j}$. It is an easy calculation using the shift operations from Proposition $A .3$ that each of the summands of $(\ddagger)$ is 1 and hence we obtain sum $r^{g}$ as expected.

We move on to a more non-trivial example. We compute the sum in $(\ddagger)$ to see that it agrees with $2^{g-1}\left(2^{g}+1\right)$ (see [B3], Section 9). Here we notice that there are three allowable $I$ 's, i.e., $\{1,2\},\{1,3\}$, and $\{1,4\}$. By a small calculation we obtain: If a summand in ( $\ddagger$ ) has $\ell\{1,3\}$ 's, then that summand is 1 if $\ell=0$ or 1 and it is $2^{\ell-1}$ otherwise. So the sum is

$$
\left(\begin{array}{l}
g \\
0
\end{array}\right) 2^{g}+\left(\begin{array}{l}
g \\
1
\end{array}\right) 2^{g-1}+\sum_{\ell=2}^{g}\left(\begin{array}{c}
g \\
\ell
\end{array}\right) 2^{\ell-1} 2^{g-\ell}=2^{g-1}\left(2^{g}+1\right) .
$$

The number $h^{0}\left(S U_{X}(r), \mathcal{L}^{k}\right) / r^{g}$ is known to be symmetric in $k$ and $r$. It is possible to see this as a consequence of Grassmann duality

$$
\operatorname{Gr}(r, r+k) \stackrel{\sim}{\rightarrow} \operatorname{Gr}(k, r+k) .
$$

The factor of $r^{g}$ in the denominator comes from the requirement that $I^{1}, \ldots, I^{g}$ in ( $)$ each contain 1 (this condition is not symmetric under Grassmann duality, but it is symmetric in a cyclic sense).

8.5. Witten's theorem and consequences. Recall that the small quantum cohomology of $\operatorname{Gr}(r, n)$ is an associative ring $\mathrm{QH}^{*}(\operatorname{Gr}(r, n))$ whose underlying abelian group is $H^{*}(\operatorname{Gr}(r, n), \mathbb{Z}) \otimes \mathbb{Z}[q]$ and the product structure is given by

$$
\omega_{I} \star \omega_{J}=\sum_{K, d}\left\langle\omega_{I}, \omega_{J}, \omega_{K}\right\rangle_{d, 0} q^{d} \omega_{K^{\prime}}
$$

(see Section A.1 for the notation) where $K$ runs through all subsets of $[n]$ of cardinality $r$ and where $d$ runs though all non-negative integers and $\omega_{K^{\prime}}$ is the dual of $\omega_{K}$; see Section A.1. We note the following formula for the (small) quantum product of Schubert classes:

$$
\omega_{I^{1}} \star \omega_{I^{2}} \star \cdots \star \omega_{I^{s}}=\sum_{K, d}\left\langle\omega_{I^{1}}, \omega_{I^{2}}, \ldots, \omega_{I^{s}}, \omega_{K}\right\rangle_{d, 0} q^{d} \omega_{K^{\prime}} .
$$

Recall that $n=r+k$. Witten's theorem [W] gives an isomorphism

$$
W: \mathrm{QH}^{*}(G r(r, n)) /(q-1) \rightarrow R(U(r))_{k, n}
$$

from the quantum cohomology of $\operatorname{Gr}(r, n)$ at $q=1$ to the Verlinde algebra of $U(r)$ at $S U(r)$ level $\leq k$ and $U(1)$ level $\leq n$. Here $R(U(r))_{k, n}$ is additively generated by sequences $\lambda=\left(\lambda_{1} \geq \lambda_{2} \geq \cdots \geq \lambda_{r}\right) \in \mathbb{Z}^{r}$ such that $\lambda_{r} \geq 0$ and $\lambda_{1} \leq k$, where $\lambda_{a}=n-r+a-i_{a}, a=1, \ldots, r$. The isomorphism $W$ takes $\omega_{I}$ to the partition $\lambda(I)$.

$R(U(r))_{k, n}$ is related to the tensor product of the Verlinde algebra $R(S U(r))_{k}$ for $S U(r)$ at level $\leq k$ and the Verlinde algebra $R(U(1))_{r n}$ of $U(1)$ at level $\leq n r$. We will make this relation precise. $R(S U(r))_{k}$ is a ring with an additive basis given by irreducible $S U(r)$ representations of level $\leq k$, and $R(U(1))_{r n}$ is generated as a ring by $x$ with relation $x^{n r}=1$.

Inside $R(S U(r))_{k} \otimes_{\mathbb{Z}} R(U(1))_{r n}$ consider the subspace $\tilde{R}$ spanned by $\bar{\lambda} \otimes x^{a}$ so that $a \equiv|\bar{\lambda}|(\bmod r)$. Clearly $\tilde{R}$ is a unital subring of $R(S U(r))_{k} \otimes R(U(1))_{r n}$. 
On $\tilde{R}$ consider the operator

$$
T\left(\bar{\lambda} \otimes x^{a}\right)=\bar{\eta} \otimes x^{n+a}
$$

where $\bar{\eta}$ is related to $\bar{\lambda}$ by the cyclic shift $\eta=\left(k+\lambda_{r} \geq \lambda_{1} \geq \lambda_{2} \geq \cdots \geq \lambda_{r-1}\right)$ and $a \equiv|\bar{\lambda}|(\bmod r)$. It is easy to check that $T(u)=u \cdot\left((k, 0, \ldots, 0) \otimes x^{n}\right)$. The $\mathbb{Z}$-submodule of $\tilde{R}$ generated by elements $T(u)-u$ for $u \in \tilde{R}$ is an ideal $I$ of $\tilde{R}$.

Lemma 8.3. (1) Each orbit $\left\{T^{b}\left(\bar{\mu} \otimes x^{a}\right)_{b=1}^{r}\right\}$ where $a \equiv|\bar{\mu}|(\bmod r)$, contains a unique element of the form $\bar{\lambda} \otimes x^{|\lambda|} \in \tilde{R}$ where the sequence $\lambda$ satisfies $\lambda_{1} \leq k$ and $\lambda_{r} \geq 0$ (and $|\lambda|=\sum_{a=1}^{r} \lambda_{a} \in \mathbb{Z}$ ).

(2) If $\bar{\mu}=0$, then the $T$ orbit of $\bar{\mu} \otimes 1$ does not contain any element of the form $\bar{\nu} \otimes 1$ with $\bar{\nu} \neq 0$.

Proof. Find a subset $K$ of $[2 n]$ so that $\bar{\lambda}(K)=\bar{\mu}$. Let $c=a-|\lambda|$. So $\bar{\mu} \otimes x^{a}=$ $\bar{\lambda}(K) \otimes x^{c+|\lambda(K)|}$. Let $J$ be the subset of $[2 n]$ obtained by subtracting 1 from elements of $K$ and replacing 0's by $n$. Then it is easy to see that $\bar{\lambda}(J) \otimes x^{c+|\lambda(J)|-r}$ equals $\bar{\lambda}(K) \otimes x^{c+|\lambda(K)|}$ if $1 \notin K$ and it equals $T\left(\bar{\lambda}(K) \otimes x^{c+|\lambda(K)|}\right)$ if $1 \in K$. Now by assumption $c \equiv 0(\bmod r)$ and hence there is a unique $\ell \in[n]$ so that $c-\ell r \equiv 0$ $(\bmod n r)$. We repeat this process $\ell$ times (the sought for $b$ is the number of elements in $K$ that are less than or equal to $\ell$ ). This proves (1). The proof of (2) follows from the definition of $T$.

The linear map $\tilde{R} \rightarrow R(U(r))_{k, n}$ sending $\bar{\lambda} \otimes x^{|\lambda|} \in \tilde{R}$ to $\lambda \in R(U(r))_{k, n}$ is a ring homomorphism with kernel $I$ and induces an isomorphism of rings

$$
\tilde{R} / I \stackrel{\sim}{\rightarrow} R(U(r))_{k, n} .
$$

Clearly elements of the form $W\left(\omega_{I}\right)$ where $I$ runs over all $r$ element subsets of $[n]$ forms an additive basis for $R(U(r))_{k, n}$.

Let $K=\{1, k+2, k+3, \ldots, k+r=n\} \subset[n]$. Then using equation (8.5) on $\lambda=(0,0, \ldots, 0) \in \mathbb{Z}^{r}$,

$$
W\left(\omega_{K}\right) x^{r}=1 \otimes 1
$$

Remark 8.4. The reader can verify the form of Witten's theorem needed in this paper by just taking $\tilde{R} / I$ to be the definition of $R(U(r))_{k, n}$ and deducing that the map $W$ is a ring homomorphism from knowing that Pieri's rule holds on both sides of equation (8.4) (using [Ber on the quantum cohomology side and $\mathrm{G}$ ] for the Verlinde algebra for $S U(r)$ at level $\leq k)$.

8.5.1. Consequence of Witten's theorem. Let $I^{1}, \ldots, I^{s}$ be subsets of $[n]$ each of cardinality $r$. Assume that there is an integer $D$ so that $\sum_{j=1}^{s} \operatorname{codim}\left(\omega_{I^{s}}\right)=r k-D r$. Then we have the following proposition which implies Proposition 8.2 immediately:

\section{Proposition 8.5.}

$$
\left\langle\omega_{I^{1}}, \omega_{I^{2}}, \ldots, \omega_{I^{s}}\right\rangle_{0, D}=N_{0}^{(k)}\left(\bar{\mu}^{1}, \ldots, \bar{\mu}^{s}\right)
$$

where $\bar{\mu}^{j}=\bar{\lambda}\left(I^{j}\right)$ are the associated representations of $S U(r)$.

Proof. For simplicity assume $D \leq 0$ (this is the case when we need this proposition). Let $K=\{1, k+2, k+3, \ldots, n\}$. We claim

$$
\left\langle\omega_{I^{1}}, \omega_{I^{2}}, \ldots, \omega_{I^{s}}\right\rangle_{0, D}=\left\langle\omega_{I^{1}}, \omega_{I^{2}}, \ldots, \omega_{I^{s}}, \omega_{K}, \omega_{K}, \ldots, \omega_{K}\right\rangle_{-D, 0}
$$

with $\omega_{K}$ repeated $-D$ times. To see this, apply the shift operation from Proposition A.3 to each of the $-D \omega_{K}$ 's appearing on the right hand side of equation (8.8). 
According to Witten's theorem the two sides of equation (8.8) equal the coefficient of $W\left(\omega_{\{1, \ldots, r\}}\right)$ in the product

$$
W\left(\omega_{K}\right)^{-D} \prod_{j=1}^{s} W\left(\omega_{I^{j}}\right) \in R(U(r))_{k, n} .
$$

First using equation (8.6), we note $W\left(\omega_{K}\right)=1 \otimes x^{r n-r}$. Also $W\left(\omega_{I^{j}}\right)=\bar{\lambda}\left(I^{j}\right) \otimes$ $x^{\operatorname{codim}\left(\omega_{I^{j}}\right)}$. Then the $U(1)$ component of the product in $\tilde{R}$ corresponds to $x$ raised to the number $(\bmod r n)$

$$
\sum_{j} \operatorname{codim}\left(I^{j}\right)+D r=r(n-r) .
$$

Therefore using Lemma $8.3(2)$, the coefficient of $W\left(\omega_{\{1, \ldots, r\}}\right)$ in

$$
W\left(\omega_{K}\right)^{-D} \prod_{j=1}^{s} W\left(\omega_{I^{j}}\right)
$$

equals the coefficient of the identity representation in the product $\prod_{j=1}^{s} \bar{\lambda}\left(I^{j}\right)$ in the Verlinde algebra of $S U(r)$ at level $\leq k$; which equals the right hand side of equation (8.7) as desired.

\section{Appendix A. Some Results on Vector Bundles on Curves AND Gromov-WitTen theory}

A.1. Schubert cycles. Given a subset $I$ of $[n]$ of cardinality $r$, we will assume that it is written in the form $\left\{i_{1}<i_{2}<\cdots<i_{r}\right\}$. Let

$$
E_{\bullet}:\{0\}=E_{0} \subsetneq E_{1} \subsetneq \cdots \subsetneq E_{n}=W
$$

be a complete flag in the $n$-dimensional vector space $W$. Define the Schubert cell $\Omega_{I}^{o}\left(E_{\bullet}\right) \subseteq \operatorname{Gr}(r, W)$ by

$$
\Omega_{I}^{o}\left(E_{\bullet}\right)=\left\{V \in \operatorname{Gr}(r, W) \mid \operatorname{rk}\left(V \cap E_{u}\right)=a \text { for } i_{a} \leq u<i_{a+1}, a=0, \ldots, r\right\}
$$

where $i_{0}$ is defined to be 0 and $i_{r+1}=n$. The cell $\Omega_{I}^{o}\left(E_{\bullet}\right)$ is smooth. Its closure will be denoted by $\Omega_{I}\left(E_{\bullet}\right)$, and the cycle class of this subvariety (in $H^{*} \operatorname{Gr}(r, W)$ ) is denoted by $\omega_{I}$. For a fixed complete flag on $W$, it is easy to see that every $r$-dimensional vector subspace belongs to a unique Schubert cell.

The dual of $\omega_{I}$ under the intersection pairing is $\omega_{I^{\prime}}$ where $I^{\prime}=\{n+1-i, i \in I\}$. This means that if $\operatorname{codim}\left(\omega_{I}\right)+\operatorname{codim}\left(\omega_{J}\right)=r(n-r)$, the intersection number $\omega_{I} \cdot \omega_{J}$ in $H^{2 r(n-r)} X=\mathbb{Z}$ is 1 if $J=I^{\prime}$ and it is 0 otherwise.

A.2. Vector bundles on curves. Let $X$ be a smooth curve of genus $g$ and let $E, F$ be vector bundles of ranks $r$ and $k$, respectively, such that $\chi(X, E \otimes F)=0$. The following lemma is due to Faltings (see e.g. $[\mathbf{S}$ ).

Lemma A.1. If $H^{0}(X, E \otimes F)=0$, then $E$ and $F$ are semi-stable.

Lemma A.2. Let $X \rightarrow S$ be a projective, flat family of curves and let $s_{0} \in S$. Suppose $E_{0}$ is a vector bundle on $X_{s_{0}}$. Then there exists an étale map $\left(T, t_{0}\right) \rightarrow$ $\left(S, s_{0}\right)$ and vector bundle $E_{T}$ on $X_{T}$ so that $E_{T, t_{0}}=E_{0}$. If $\operatorname{det}\left(E_{0}\right)$ is trivial, then there exist such $\left(T, t_{0}, E_{T}\right)$ so that $\operatorname{det}\left(E_{T}\right)$ is trivial. 
Proof (Standard; for example, see [SGA], Proposition 4.1, page 46). We need to extend $E_{0}$ as a vector bundle. We will extend formally first, over successive thickenings of the central fibers. Assume that $E$ has been lifted to $\mathcal{X}_{A} \rightarrow \operatorname{Spec} A$ (the base change of $X \rightarrow S$ to Spec $A$ ) and that $B$ is an Artinian local ring with an ideal $I$ such that $I^{2}=0$ and $B / I=A$. We want to extend $E$ to $\mathcal{X}_{B} \rightarrow \operatorname{Spec}(B)$. This is clear because (formally) the obstruction to the extension over Artin local rings is in $H^{2}\left(\mathcal{X}_{A}, M_{n}(I)\right)(n \times n$ matrices with entries in $I)$ which vanishes on a curve. We now apply Artin approximation theorem $\mathrm{Ar}$ to obtain $\left(T, t_{0}\right)$.

If $\operatorname{det}\left(E_{0}\right)$ is trivial and we want the extension to preserve this, the extension problem over successive Artin rings is controlled by $H^{2}$ of traceless matrices with coefficients in $I$, which is again 0 .

A.3. Shift operations in Gromov-Witten theory. Let $I^{1}, \ldots, I^{s}$ be subsets of $[n]$ each of cardinality $r$ and let $d, D$ be integers. Suppose $I^{1}=\left\{i_{1}<\cdots<i_{r}\right\}$. Define $J$ a subset of $[n]$ of cardinality $r$ and an integer $\tilde{d}$ as follows:

(1) if $i_{1}>1$, let $J=\left\{i_{1}-1<\cdots<i_{r}-1\right\}$ and $\tilde{d}=d$,

(2) if $i_{1}=1$, let $J=\left\{i_{2}-1<\cdots<i_{r}-1<n\right\}$ and $\tilde{d}=d-1$.

The following proposition is proved in Be2] (Proposition 2.5).

\section{Proposition A.3.}

$$
\left\langle\omega_{I^{1}}, \omega_{I^{2}}, \ldots, \omega_{I^{s}}\right\rangle_{d, D}=\left\langle\omega_{J}, \omega_{I^{2}}, \ldots, \omega_{I^{s}}\right\rangle_{\tilde{d}, D-1} .
$$

We recall the reason for the equality (see $\mathrm{Be} 2$ for more details). We first verify that the expected dimensions of both the intersections in the above equality of Gromov-Witten numbers are the same. Let $\mathrm{S}=\left\{\rho_{1}, \ldots, \rho_{s}\right\}$ be a set of (distinct points) on $\mathbb{P}^{1}$ as before. Let $T$ be an ES-bundle of degree $-D$ and rank $n$. Choose generic complete flags $E_{\bullet}^{\rho_{j}}$ on the fibers $T_{\rho_{j}}$ for $j=1, \ldots, s$.

Let $t$ be a uniformizing parameter at $\rho_{1} \in \mathbb{P}^{1}$. Define $\tilde{T}$ to be the vector bundle which agrees with $T$ in $\mathbb{P}^{1}-\left\{\rho_{1}\right\}$ and whose sections in a small neighborhood $U$ of $\rho_{1}$ are sections $s$ of $T$ on $U-\left\{\rho_{1}\right\}$ such that $t s$ is a holomorphic section of $T$ on $U$ whose fiber at $\rho_{1}$ lies in $E_{1}^{\rho_{1}}$ (the first element of the flag). As coherent sheaves, $\tilde{T} \supset T$. It can be shown that $\tilde{T}$ is ES as well.

$\tilde{T}$ inherits complete flags from $T$ on its fibers at each point of $\left\{\rho_{1}, \ldots, \rho_{s}\right\}$. There is a bijection between the set of subbundles of $T$ and those of $\tilde{T}$ both restricted to $\mathbb{P}^{1}-\left\{\rho_{1}\right\}$. Proposition A.3 follows from this bijection and a calculation at $\rho_{1}$ (this gives an inequality between the two Gromov-Witten numbers in Proposition A.3 to start with, but this is a cyclic process so we obtain equality).

\section{ACKNOWLEDGMENTS}

I thank A. Boysal, P. Brosnan, M. V. Nori, and M. Popa for useful discussions. I acknowledge help from Mihnea Popa in correcting an initial naive idea.

\section{REFERENCES}

[Ab] T. Abe, On SL(2) - GL( $n)$ strange duality. Preprint.

[A] S. Agnihotri. Quantum Cohomology and the Verlinde Algebra. Ph.D thesis, University of Oxford, 1995.

[Ar] M. Artin. Algebraic approximation of structures over complete local rings. Inst. Hautes Études Sci. Publ. Math., No. 36, 1969, 23-58. MR0268188 (42:3087) 
[B1] A. Beauville. Fibrés de rang deux sur une courbe, fibré déterminant et fonctions thêta. II. Bull. Soc. Math. France 119 (1991), no. 3, 259-291. MR1125667 (92m:14041)

[B2] A. Beauville. Vector bundles on curves and generalized theta functions: recent results and open problems. Current topics in complex algebraic geometry (Berkeley, CA, 1992/93), 1733. Math. Sci. Res. Publ., 28. Cambridge university Press, Campridge, 1995. MR1397056 (97h:14015)

[B3] A. Beauville. Vector bundles on Riemann surfaces and Conformal Field Theory. Algebraic and Geometric Methods in Mathematical Physics, 145-166, Kluwer (1996). MR 1385680 (97e:14016)

[BL] A. Beauville, Y. Laszlo. Conformal blocks and generalized theta functions. Comm. Math. Phys. 164 (1994), no. 2, 385-419. MR1289330(95k:14011)

[BNR] A. Beauville, M. S. Narasimhan and S. Ramanan. Spectral curves and the generalised theta divisor, J. Reine Agnew. Math. 398 (1989), 169-179. MR.998478 (91c:14040)

[Be1] P. Belkale. Invariant theory of $\mathrm{GL}(n)$ and intersection theory of Grasmannians. International Math. Research Notices, Vol. 2004, no. 69, 3709-3721. MR2099498 (2005h:14117)

[Be2] P. Belkale. Quantum generalization of the Horn conjecture. Preprint, math.AG/0303013.

[Ber] A. Bertram. Quantum Schubert Calculus. Advances in Math. 128 (1997), no. 2, 289-305. MR $1454400(98 \mathrm{j}: 14067)$

[BL] M. Brion, V. Lakshmibai. A geometric approach to standard monomial theory. Represent. Theory 7 (2003), 651-680. MR2017071 (2004m:14106)

[BP] M. Brion, P. Polo. Large Schubert varieties. Represent. Theory 4 (2000), 97-126. MR.1789463 (2001j:14066)

[SGA] P. Deligne. Cohomologie étale. Séminaire de Géométrie Algébrique du Bois-Marie. Lecture Notes in Mathematics, Vol. 569. Springer-Verlag, Berlin-New York, 1977. MR0463174 $(57: 3132)$

[DT] R. Donagi, L. Tu. Theta functions for SL $(n)$ versus GL( $n)$. Math. Res. Lett. 1 (1994), no. 3, 345-357. MR.1302649 (95j:14012)

[DN] J. M. Drezet, M.S. Narasimhan. Groupe de Picard des variétés de modules de fibrés semistables sur les courbes algébriques. Invent. Math. 97 (1989), no. 1, 53-94. MR999313 (90d:14008)

[E] E. Esteves. Separation properties of theta functions. Duke Math. J. 98 (1999), no. 3, 565593. MR 1695802 (2000e:14048)

[Fa1] G. Faltings. Stable $G$-bundles and projective connections. J. Algebraic Geom. 2 (1993), no. 3, 507-568. MR.1211997 (94i:14015)

[Fa2] G. Faltings. A proof for the Verlinde formula. J. Algebraic Geom. 3 (1994), no. 2, 347-374. MR.1257326 (95j:14013)

[Fu] W. Fulton. Intersection Theory. Springer-Verlag, Berlin, 1998. MR1644323 (99d:14003)

[FP] W. Fulton, R. Pandharipande. Notes on stable maps and quantum cohomology. Algebraic geometry-Santa Cruz 1995, 45-96, Proc. Sympos. Pure Math., 62, Part 2, Amer. Math. Soc., Providence, RI, 1997. MR1492534 (98m:14025)

[G] D. Gepner. Fusion rings and geometry. Comm. Math. Phys. 141, no. 2 (1991), 381-411. MR.1133272 (92j:81271)

[Kl] S.L. Kleiman. The transversality of a general translate. Compositio Math. 38 (1974), 287297. MR0360616 (50:13063)

[KM] F. Knudsen, D. Mumford. The projectivity of the moduli space of stable curves I. Preliminaries on "det" and "div". Math. Scand. 39 (1976), 19-55. MR0437541 (55:10465)

[KNR] S. Kumar, M. S. Narasimhan, A. Ramanathan. Infinite Grassmannians and moduli spaces of $G$-bundles. Math. Ann. 300 (1994), no. 1, 41-75. MR.1289830(96e:14011)

[L] Y. Laszlo, Á propos de l'espace des modules de fibrés de rang 2 sur une courbe. Math. Ann. 299 (1994), no. 4, 597-608. MR1286886 (95f:14021)

[Mi] J. S. Milne. Étale cohomology. Princeton Mathematical Series, 33. Princeton University Press, Princeton, N.J., 1980. MR.559531 (81j:14002)

[Pa] C. Pauly, Espaces de modules de fibrés paraboliques et blocs conformes. Duke Math. J. 84 (1996), no. 1, 217-235. MR.1394754 (97h:14022)

[Po] A. Polishchuk. Abelian Varieties, Theta Functions and the Fourier Transform. Cambridge Tracts in Mathematics, 153. MR 1987784 (2004m:14094)

[P] M. Popa. Verlinde bundles and generalized theta linear series. Trans. Amer. Math. Soc. 354 (2002), no. 5, 1869-1898. MR1881021(2003i:14046) 
[Pot] J. Le Potier. Lectures on vector bundles. Cambridge Studies in Advanced Mathematics, 54. MR 1428426 (98a:14019)

[S] C. S. Seshadri. Vector bundles on curves. Linear algebraic groups and their representations (editors: Elman, Schacher, Varadarajan), Contemporary Math., 153. MR1247504 (95b:14008)

[TUY] A. Tsuchiya, K. Ueno, Y. Yamada. Conformal field theory on universal family of stable curves with gauge symmetries. Integrable systems in quantum field theory and statistical mechanics, 459-566. Adv. Stud. Pure Math., 19, Academic Press, Boston, MA, 1989. MR.1048605 (92a:81191)

[vGP] B. van Geemen, E. Previato. Prym varieties and the Verlinde formula. Math. Ann. 294 (1992), no. 4, 741-754. MR.1190454 (93j:14037)

[W] E. Witten. The Verlinde algebra and the cohomology of the Grassmannian. Geometry, topology and physics, 357-422, Conf. Proc. Lecture Notes Geom. Topology, IV, Internat. Press, Cambridge, MA, 1995. MR:1358625 (98c:58016)

Department of Mathematics, University of North Carolina-Chapel Hill, CB \#3250, Phillips Hall, Chapel Hill, North Carolina 27599

E-mail address: belkale@email.unc.edu 